\title{
A Galerkin method for retarded boundary integral equations with smooth and compactly supported temporal basis functions
}

\author{
Sauter, Stefan ; Veit, Alexander
}

\begin{abstract}
We consider retarded boundary integral formulations of the three-dimensional wave equation in unbounded domains. Our goal is to apply a Galerkin method in space and time in order to solve these problems numerically. In this approach the computation of the system matrix entries is the major bottleneck. We will propose new types of finite-dimensional spaces for the time discretization. They allow variable time-stepping, variable order of approximation and simplify the quadrature problem arising in the generation of the system matrix substantially. The reason is that the basis functions of these spaces are globally smooth and compactly supported. In order to perform numerical tests concerning our new basis functions we consider the special case that the boundary of the scattering problem is the unit sphere. In this case explicit solutions of the problem are available which will serve as reference solutions for the numerical experiments.
\end{abstract}

DOI: https://doi.org/10.1007/s00211-012-0483-7

Posted at the Zurich Open Repository and Archive, University of Zurich

ZORA URL: https://doi.org/10.5167/uzh-87098

Journal Article

Published Version

Originally published at:

Sauter, Stefan; Veit, Alexander (2013). A Galerkin method for retarded boundary integral equations with smooth and compactly supported temporal basis functions. Numerische Mathematik, 123(1):145-176.

DOI: https://doi.org/10.1007/s00211-012-0483-7 


\title{
A Galerkin method for retarded boundary integral equations with smooth and compactly supported temporal basis functions
}

\author{
S. Sauter - A. Veit
}

Received: 2 March 2011 / Revised: 6 March 2012 / Published online: 27 June 2012

(C) Springer-Verlag 2012

\begin{abstract}
We consider retarded boundary integral formulations of the threedimensional wave equation in unbounded domains. Our goal is to apply a Galerkin method in space and time in order to solve these problems numerically. In this approach the computation of the system matrix entries is the major bottleneck. We will propose new types of finite-dimensional spaces for the time discretization. They allow variable time-stepping, variable order of approximation and simplify the quadrature problem arising in the generation of the system matrix substantially. The reason is that the basis functions of these spaces are globally smooth and compactly supported. In order to perform numerical tests concerning our new basis functions we consider the special case that the boundary of the scattering problem is the unit sphere. In this case explicit solutions of the problem are available which will serve as reference solutions for the numerical experiments.
\end{abstract}

Mathematics Subject Classification $35 \mathrm{~L} 05 \cdot 65 \mathrm{~N} 38 \cdot 65 \mathrm{R} 20$

\section{Introduction}

Mathematical modeling of acoustic and electromagnetic wave propagation and its efficient and accurate numerical simulation is a key technology for numerous engineering applications as, e.g., in detection (non-destructive testing, radar), communication

A. Veit gratefully acknowledges the support given by SNF, No. PDFMP2_127437/1.

S. Sauter $(\varangle) \cdot$ A. Veit

Institut für Mathematik, Universität Zürich, Winterthurerstr 190, 8057 Zurich, Switzerland

e-mail: stas@math.uzh.ch

A. Veit

e-mail: alexander.veit@math.uzh.ch 
(optoelectronic and wireless) and medicine (sonic imaging, tomography). An adequate model problem for the development of efficient numerical methods for such types of physical applications is the three-dimensional wave equation in unbounded exterior domains. In this setting the method of integral equations is an elegant approach since it reduces the problem in the unbounded domain to an integral equation on the bounded surface of the scatterer.

In this paper we apply a Galerkin method for the discretization of these retarded boundary integral equations (cf. [3,14,18,19]). This approach allows variable time stepping and spatially curved scatterers. Applications where variable time stepping becomes important include problems with non-compatible Dirichlet-data at time $t=0$ and/or scatterers with very non-uniform eccentricities. Until now, a severe drawback of this method was, however, that the domain for the spatial integration is the intersection of (possibly curved) pairs of surface panels with the discrete light cone which is very complicated to handle numerically. Quadrature schemes tailored to this problem were derived for example in $[16,22,26]$. These methods are restricted to polyhedral scatterers and their implementation is difficult.

Other approaches for the numerical discretization of retarded boundary integral equations use collocation schemes (cf. [8,9,12,15,23]). Although they play an important role in practice, the mathematical analysis of these methods is challenging. In more than two dimensions stability and convergence of collocation schemes can only be shown for special geometries (cf. [13]). Furthermore the application of these techniques to curved scatterers is difficult. More recent approaches include methods based on bandlimited interpolation and extrapolation (cf. [31-34]) and convolution quadrature (cf. [4-7, 10,20,21,30]). The latter enjoys nice stability properties and allows to apply many techniques known from frequency domain problems. However the stepsize for the time discretization must be constant in these methods and a generalization to non-uniform time meshes is not straightforward.

In our paper we will present a new time discretization method for the retarded potential equations which circumvents the numerical integration over intersections of the light cone with the spatial surface mesh. For this purpose, we will introduce infinitely smooth and compactly supported basis functions in time. These functions are constructed by using the partition of unity method (PUM) (cf. [2]).

In order to test the choice of the new basis functions numerically we consider the wave equation on the sphere with Dirichlet boundary conditions. For the resulting problems explicit representations of the exact solutions are available (cf. [25]). We apply a Galerkin method using our basis functions to these problems and perform numerical experiments.

\section{Integral formulation of the wave equation}

Let $\Omega \subset \mathbb{R}^{3}$ be a Lipschitz domain with boundary $\Gamma$. We consider the homogeneous wave equation

$$
\partial_{t}^{2} u-\Delta u=0 \text { in } \Omega \times[0, T]
$$

with initial conditions

$$
u(\cdot, 0)=\partial_{t} u(\cdot, 0)=0 \text { in } \Omega
$$


and Dirichlet boundary conditions

$$
u=g \quad \text { on } \Gamma \times[0, T]
$$

on a time interval $[0, T]$ for $T>0$. In applications, $\Omega$ is often the unbounded exterior of a bounded domain. For such problems, the method of boundary integral equations is an elegant tool where this partial differential equation is transformed to an equation on the bounded surface $\Gamma$. We employ an ansatz as a single layer potential for the solution $u$,

$$
u(x, t):=S \phi(x, t):=\int_{\Gamma} \frac{\phi(y, t-\|x-y\|)}{4 \pi\|x-y\|} d \Gamma_{y}, \quad(x, t) \in \Omega \times[0, T]
$$

with unknown density function $\phi . S$ is also referred to as retarded single layer potential due to the retarded time argument $t-\|x-y\|$ which connects time and space variables.

The ansatz (2.2) satisfies the wave equation (2.1a) and the initial conditions (2.1b). Since the single layer potential can be extended continuously to the boundary $\Gamma$, the unknown density function $\phi$ is determined such that the boundary conditions (2.1c) are satisfied. This results in the boundary integral equation for $\phi$,

$$
\int_{\Gamma} \frac{\phi(y, t-\|x-y\|)}{4 \pi\|x-y\|} d \Gamma_{y}=g(x, t) \quad \forall(x, t) \in \Gamma \times[0, T] .
$$

In order to solve this boundary integral equation numerically we introduce the following space-time variational formulation (cf. $[3,18]$ ): find $\phi$ such that

$$
\int_{0}^{T} \int_{\Gamma} \int_{\Gamma} \frac{\dot{\phi}(y, t-\|x-y\|) \zeta(x, t)}{4 \pi\|x-y\|} d \Gamma_{y} d \Gamma_{x} d t=\int_{0}^{T} \int_{\Gamma} \dot{g}(x, t) \zeta(x, t) d \Gamma_{x} d t
$$

for all $\zeta$, where we denote by $\dot{\phi}$ the derivative with respect to time.

\section{Numerical discretization}

We turn our attention to the discretization of (2.4). In order to find an approximate solution we apply a Galerkin method in space and time. The variational formulation (2.4) is coercive in

$$
H^{-1 / 2,-1 / 2}(\Gamma \times[0, T]):=L^{2}\left(0, T ; H^{-1 / 2}(\Gamma)\right)+H^{-1 / 2}\left(0, T ; L^{2}(\Gamma)\right)
$$

(cf. [18]) and is uniquely solvable in this Sobolev space. Furthermore this ensures existence and uniqueness of the solution of a conforming Galerkin discretization.

Let $V_{\text {Galerkin }}$ be a finite dimensional subspace of (3.1) being spanned by $N$ basis functions $\left\{b_{i}\right\}_{i=1}^{N}$ in time and $M$ basis functions $\left\{\varphi_{i}\right\}_{i=1}^{N}$ in space. This leads to the ansatz 


$$
\phi_{\text {Galerkin }}(x, t)=\sum_{i=1}^{N} \sum_{j=1}^{M} \alpha_{i}^{j} \varphi_{j}(x) b_{i}(t), \quad(x, t) \in \Gamma \times[0, T],
$$

where $\alpha_{i}^{j}$ are the unknown coefficients. Plugging the ansatz (3.2) into the variational formulation leads to the Galerkin discretization: find $\alpha_{i}^{j}, i=1, \ldots, N, j=1, \ldots, M$ such that

$$
\begin{aligned}
& \int_{0}^{T} \int_{\Gamma} \int_{\Gamma} \sum_{i=1}^{N} \sum_{j=1}^{M} \frac{\alpha_{i}^{j} \varphi_{j}(y) \dot{b}_{i}(t-\|x-y\|) \varphi_{l}(x) b_{k}(t)}{4 \pi\|x-y\|} d \Gamma_{y} d \Gamma_{x} d t \\
& =\int_{0}^{T} \int_{\Gamma} \dot{g}(x, t) \varphi_{l}(x) b_{k}(t) d \Gamma_{x} d t
\end{aligned}
$$

for $k=1, \ldots, N$ and $l=1, \ldots, M$. A convergence analysis of this Galerkin approach using piecewise polynomial basis functions in space and time is given in [3].

Rearranging terms shows that the above formulation is equivalent to: find $\alpha_{i}^{j}$ for $i=1 \ldots, N$ and $j=1, \ldots, M$ such that

$$
\sum_{i=1}^{N} \sum_{j=1}^{M} A_{j, l}^{i, k} \alpha_{i}^{j}=g_{l}^{k} \quad \forall 1 \leq k \leq N \quad \forall 1 \leq l \leq M
$$

where

$$
g_{l}^{k}:=\int_{0}^{T} \int_{\Gamma} \dot{g}(x, t) \varphi_{l}(x) b_{k}(t) d \Gamma_{x} d t
$$

and

$$
\begin{aligned}
A_{j, l}^{i, k}: & =\int_{\Gamma} \int_{\Gamma} \varphi_{j}(y) \varphi_{l}(x) \psi_{i, k}(\|x-y\|) d \Gamma_{y} d \Gamma_{x} \\
& =\int_{\operatorname{supp}\left(\varphi_{l}\right)} \int_{\operatorname{supp}\left(\varphi_{j}\right)} \varphi_{j}(y) \varphi_{l}(x) \psi_{i, k}(\|x-y\|) d \Gamma_{y} d \Gamma_{x}
\end{aligned}
$$

with

$$
\psi_{i, k}(r):=\int_{0}^{T} \frac{\dot{b}_{i}(t-r) b_{k}(t)}{4 \pi r} d t
$$

where $r \in \mathbb{R}_{>0}$. The computation of a Galerkin solution via (3.3) leads to a large linear system with $N M$ unknowns. The corresponding boundary element matrix consists of 
$N \times N$ blocks of size $M \times M$. Each matrix block is symmetric and furthermore sparse if the basis functions in space and time have compact support (cf. [25]). This is due to the fact that $\psi_{i, k}$ has compact support in this case and therefore only those combinations of $j$ and $l$ lead to non-zero matrix entries for which

$$
\left\{\|x-y\|, x \in \operatorname{supp}\left(\varphi_{l}\right), y \in \operatorname{supp}\left(\varphi_{j}\right)\right\} \cap \operatorname{supp}\left(\psi_{i, k}\right) \neq \emptyset .
$$

The numerical realization of the Galerkin method requires the efficient and accurate approximation of the matrix entries $A_{j, l}^{i, k}$ which is a major challenge. In the literature (cf. $[18,19,26]$ ) piecewise polynomial basis functions in time are employed while, then, $\psi_{i, k}(\|x-y\|)$ in general is only a piecewise analytic function in $x \in \operatorname{supp}\left(\varphi_{l}\right)$ and $y \in \operatorname{supp}\left(\varphi_{j}\right)$ (even if $\operatorname{supp}\left(\varphi_{l}\right)$ and $\operatorname{supp}\left(\varphi_{j}\right)$ are properly separated). Consequently, high order Gauss rules are converging only at a suboptimal rate. To obtain a sufficiently high accuracy, the integration is carried out on the intersections of the surface panels with the discrete light cone, i.e., with the support of $\psi_{i, k}(\|x-y\|)$. The stable handling of these intersections and the implementation of these quadrature rules is difficult and especially complicated for curved surface patches.

In this paper, we will introduce infinitely smooth and compactly supported basis functions in time. This will simplify the problem of computing the matrix entries $A_{j, l}^{i, k}$ considerably while maintaining the sparsity of the system matrix. Since the integrand will be smooth in this case we can apply standard quadrature rules to the double integral in (3.4). Furthermore the discretization with curved surface panels is straightforward since the numerical handling of the complicated geometry of the intersection of panels with the discrete light cone is circumvented.

The basis functions in time that we will construct here, will not lead to a lower triangular Toeplitz system as standard schemes using piecewise polynomial basis functions and equidistant time grids. In our case the boundary element matrix will be a blockmatrix where the lower triangular part in general is non-zero and also a few off-diagonals are non-vanishing. Therefore FFT-type methods for Toeplitz matrices cannot be used for this type of matrices-instead, efficient iterative methods have to be employed (and, firstly, developed). We expect that for certain classes of applications, e.g., for problems with non-compatible Dirichlet data, the savings by using substantially less (variable) timesteps compared to uniform time stepping are significant and lead to a faster algorithm.

The construction of the aforementioned basis functions in time is in the spirit of the PUM (cf. [2]). Before we define and construct the finite element space in time we recall some basic definitions of the PUM.

Definition 3.1 Let $\Theta:=[0, T]$ be the time interval and $\left\{\Theta_{i}\right\}$ be a closed cover of $\Theta$ satisfying the overlap condition

$$
\exists L \in \mathbb{N} \quad \text { s.t } \quad \forall t \in \Theta, \quad \#\left\{i \mid t \in \Theta_{i}\right\} \leq L .
$$

Let $\left\{\varphi_{i}\right\} \subset C^{m}(\mathbb{R}), m \in \mathbb{N}_{0}$ be a partition of unity subordinate to the cover $\left\{\Theta_{i}\right\}$ with 


$$
\begin{aligned}
& \operatorname{supp} \varphi_{i} \subset \Theta_{i}, \quad \sum_{i} \varphi_{i} \equiv 1 \text { on } \Theta \\
& \left\|\varphi_{i}\right\|_{L^{\infty}(\mathbb{R})} \leq C_{\infty},\left\|\varphi_{i}^{\prime}\right\|_{L^{\infty}(\mathbb{R})} \leq \frac{C_{G}}{\left|\Theta_{i}\right|},
\end{aligned}
$$

for all $i$ where $C_{\infty}$ and $C_{G}$ are constants and $\left|\Theta_{i}\right|$ denotes the length of the interval $\Theta_{i}$. Then $\left\{\varphi_{i}\right\}$ is called a $\left(L, C_{\infty}, C_{G}\right)$ partition of unity of degree $m$ subordinate to the cover $\left\{\Theta_{i}\right\}$.

Multiplying such a partition of unity with localized finite dimensional spaces $S_{i}$ consisting of functions with support in $\Theta_{i}$ leads to PUM spaces on $[0, T]$.

Definition 3.2 Let $\Theta$ and $\left\{\Theta_{i}\right\}$ be as in Definition 3.1 and let $\left\{\varphi_{i}\right\}$ be a $\left(L, C_{\infty}, C_{G}\right)$ partition of unity subordinate to $\left\{\Theta_{i}\right\}$. Let $S_{i} \subset\left\{w \in L^{2}(\Theta)\right.$ : supp $\left.w \in \Theta_{i}\right\}$ be given. Then the space

$$
S:=\sum_{i} \varphi_{i} S_{i}:=\left\{\sum_{i} \varphi_{i} v_{i} \mid v_{i} \in S_{i}\right\} \subset L^{2}(\Theta)
$$

is called the PUM space. The spaces $S_{i}$ are the local approximation spaces.

In Definition 3.2, $S$ is a subspace of $L^{2}(\Theta)$. We can easily obtain smoother spaces by choosing an appropriate partition of unity and smooth local approximation spaces. As mentioned above our goal is to define a PUM space $S \subset C^{\infty}(\mathbb{R})$ with smooth and compactly supported basis functions. Therefore we will first construct a partition of unity of infinite degree. Consider the function ${ }^{1}$

$$
f(t):= \begin{cases}\operatorname{erf}(2 \operatorname{arctanh}(t)), & \text { for }|t|<1 \\ -1, & \text { for } t \leq-1 \\ 1, & \text { for } t \geq 1\end{cases}
$$

Lemma 3.3 The function $f$ as defined in (3.5) belongs to $C^{\infty}(\mathbb{R})$.

Proof It can be proved by induction that the $m$ th derivative of $f$ in the interval $(-1,1)$ can be written as

$$
f^{(m)}(t)=C \mathrm{e}^{-4 \operatorname{arctanh}^{2}(t)}\left(t^{2}-1\right)^{-m} \sum_{i=0}^{m-1} \alpha_{i} \operatorname{arctanh}^{i}(t) t^{m-i-1}
$$

for constants $C$ and $\alpha_{i}$. Therefore

$$
\lim _{|t| \rightarrow 1} f^{(m)}(t)=0
$$

for arbitrary $m \in \mathbb{N}$.

\footnotetext{
${ }^{1}$ Note that this choice of $f$ is by no means unique. In [11, Sec. 6.1], $C^{\infty}(\mathbb{R})$ bump functions are considered (in a different context) which have certain Gevrey regularity. They also could be used for our partition of unity.
} 
Let $a<b$ be two real numbers. We make a change of variable and define

$$
h_{a, b}(t):=\frac{1}{2} f\left(2 \frac{t-a}{b-a}-1\right)+\frac{1}{2} .
$$

Then $h_{a, b}: \mathbb{R} \rightarrow[0,1]$ is a $C^{\infty}$-function such that

$$
h_{a, b}(t)= \begin{cases}0, & \text { for } t \leq a \\ 1, & \text { for } t \geq b\end{cases}
$$

Now we can define a $C^{\infty}$-bump function $\rho_{a, b, c}$ for real numbers $a<b<c$ by

$$
\rho_{a, b, c}(t):= \begin{cases}h_{a, b}(t), & \text { for } t \leq b \\ 1-h_{b, c}(t), & \text { for } t \geq b\end{cases}
$$

Due to the above properties, $\rho_{a, b, c}$ satisfies $\rho_{a, b, c} \geq 0$ in $\mathbb{R}$ and

$$
\rho_{a, b, c}(t)= \begin{cases}0, & \text { for } t \leq a \text { and } t \geq c, \\ 1, & \text { for } t=b\end{cases}
$$

Let us now consider the closed interval $\Theta=[0, T]$ and $N$ (not necessarily equidistant) timesteps $t_{i}$ such that $0=t_{0}<t_{1}<t_{2}<\cdots<t_{N-2}<t_{N-1}=T$. We define $\tau_{i}:=\left[t_{i-1}, t_{i}\right]$ for $i=1, \ldots, N-1$. Then a closed cover $\left\{\Theta_{i}\right\}$ of $\Theta$, satisfying the pointwise overlap condition in Definition 3.1 with $L=2$, is given by

$$
\begin{aligned}
\Theta_{1} & :=\tau_{1}, \\
\Theta_{i} & :=\tau_{i-1} \cup \tau_{i} \text { for } i=2, \ldots, N-1, \\
\Theta_{N} & :=\tau_{N-1} .
\end{aligned}
$$

Next we define

$$
\begin{aligned}
\varphi_{1}(t) & :=1-h_{t_{0}, t_{1}}(t) \\
\varphi_{i}(t) & :=\rho_{t_{i-2}, t_{i-1}, t_{i}}(t) \text { for } i=2, \ldots, N-1, \\
\varphi_{N}(t) & :=h_{t_{N-2}, t_{N-1}}(t) .
\end{aligned}
$$

Then $\left\{\varphi_{i}\right\}$ is a smooth partition of unity subordinate to the cover $\left\{\Theta_{i}\right\}$. Figure 1 shows an example of such a set of functions.

We want a more detailed characterization of this partition of unity in the sense of Definition 3.1 in order to get error estimates for the PUM. Therefore we assume that the partition is locally quasiuniform:

$$
1 \leq \frac{\left|\Theta_{i}\right|}{\min \left\{\left|\tau_{i-1}\right|,\left|\tau_{i}\right|\right\}} \leq c_{\max } \text { for } i=2, \ldots, N-1
$$




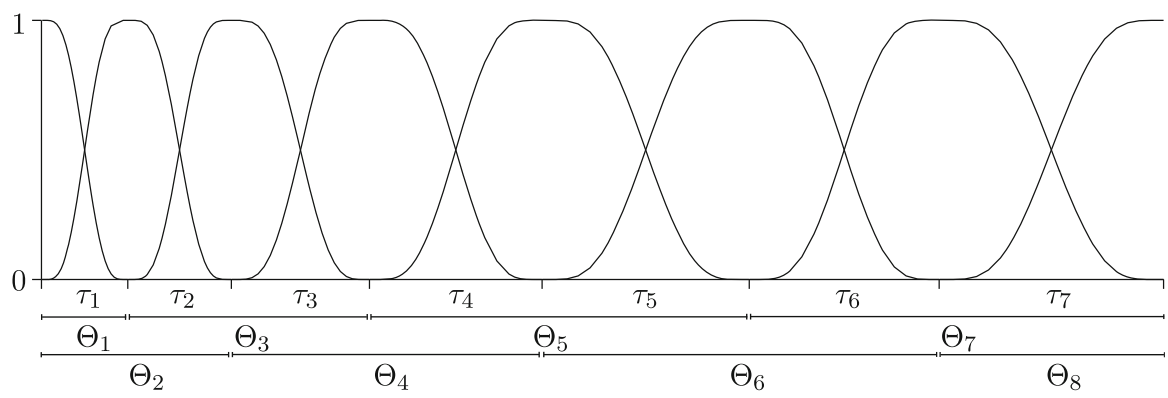

Fig. 1 Partition of unity $\left\{\varphi_{i}\right\}$ subordinate to the cover $\left\{\Theta_{i}\right\}$ for $N=4$

with a moderate constant $c_{\max }$. By taking into account

$$
\left\|h_{a, b}^{\prime}\right\|_{L^{\infty}(\mathbb{R})}=\left|h_{a, b}^{\prime}\left(\frac{a+b}{2}\right)\right|=\frac{4 \pi^{-1 / 2}}{b-a}
$$

we get

$$
\begin{aligned}
\left\|\varphi_{1}^{\prime}\right\|_{L^{\infty}([0, T])} & =\frac{4 \pi^{-1 / 2}}{\left|\Theta_{1}\right|}, \\
\left\|\varphi_{i}^{\prime}\right\|_{L^{\infty}([0, T])} & =\frac{4 \pi^{-1 / 2}}{\min \left\{\left|\tau_{i-1}\right|,\left|\tau_{i}\right|\right\}} \leq \frac{4 \pi^{-1 / 2} c_{\max }}{\left|\Theta_{i}\right|} \text { for } i=2, \ldots, N-1, \\
\left\|\varphi_{N}^{\prime}\right\|_{L^{\infty}([0, T])} & =\frac{4 \pi^{-1 / 2}}{\left|\Theta_{N}\right|} .
\end{aligned}
$$

Since $\left\|\varphi_{i}\right\|_{L^{\infty}([0, T])}=1$ for $i=1, \ldots, N$ we get that $\left\{\varphi_{i}\right\}$ is a $\left(2,1,4 \pi^{-1 / 2} c_{\max }\right)$ partition of unity of infinite degree subordinate to the cover $\left\{\Theta_{i}\right\}$.

With this construction of a smooth and compactly supported partition of unity we will define the global finite element space according to Definition 3.2. By taking into account that the exact solution of (2.1a)-(2.1c) and its derivative vanish at $t=0$ we define, for given polynomial degree $p \in \mathbb{N}$, the spaces

$$
\begin{aligned}
S_{1} & :=t^{2} \mathbb{P}_{p-2} \text { on } \Theta_{1}, \\
S_{i} & :=\mathbb{P}_{p} \text { on } \Theta_{i}, \quad i=2, \ldots, N,
\end{aligned}
$$

where $\mathbb{P}_{p}$ denotes the space of polynomials of degree $p$ and, formally, we set $\mathbb{P}_{-2}:=$ $\mathbb{P}_{-1}:=\mathbb{P}_{0}$.

Remark 3.4 The definition of the spaces $S_{i}$ could be generalized by choosing local polynomial degrees $p_{i}$ depending on the local patches $\Theta_{i}$ in the spirit of adaptive $h p$ methods. We do not elaborate on this aspect here.

The global PUM space $S$ contains linear combinations of products of polynomials and functions of the partition of unity $\left\{\varphi_{i}\right\}$. To derive error estimates for the PUM 
we remark that the spaces $S_{i}$ meet the following approximation property: let $u \in$ $H^{k}(\Theta), k \geq 1$. Then, for each patch $\Theta_{i}, 1 \leq i \leq N$, there exists $u_{S_{i}} \in S_{i}$ such that

$$
\begin{aligned}
\left\|u-u_{S_{i}}\right\|_{L^{2}\left(\Theta_{i}\right)} & \leq C_{1}\left|\Theta_{i}\right|^{\min (k-1, p)+1}\|u\|_{H^{k}\left(\Theta_{i}\right)}, \\
\left\|u^{\prime}-u_{S_{i}}^{\prime}\right\|_{L^{2}\left(\Theta_{i}\right)} & \leq C_{2}\left|\Theta_{i}\right|^{\min (k-1, p)}\|u\|_{H^{k}\left(\Theta_{i}\right)},
\end{aligned}
$$

where $C_{1}$ and $C_{2}$ depend on $k, p$ and $c_{\max }$. From [2, Theorem 1] we conclude that the global approximation

$$
u_{S}=\sum_{i=1}^{N} \varphi_{i} u_{S_{i}} \in S \subset H^{1}(\Theta)
$$

satisfies the error bounds

$$
\begin{aligned}
\left\|u-u_{S}\right\|_{L^{2}(\Theta)} & \leq 2 C_{1} \tilde{\Theta}^{\min (k-1, p)+1}\|u\|_{H^{k}(\Theta)}, \\
\left\|u^{\prime}-u_{S}^{\prime}\right\|_{L^{2}(\Theta)} & \leq 2 C_{2} \sqrt{8 \pi^{-1 / 2} c_{\max }+2} \tilde{\Theta}^{\min (k-1, p)}\|u\|_{H^{k}(\Theta)} .
\end{aligned}
$$

where $\tilde{\Theta}:=\max _{1 \leq i \leq N}\left|\Theta_{i}\right|$. For the implementation of this method we need a basis of the PUM space. It can be determined by multiplying the basis elements of the local approximation spaces with the appropriate partition of unity function. An $L^{2}(-1,1)$ orthogonal basis of $\mathbb{P}_{p}$ is given by the Legendre polynomials $\left\{P_{m}\right\}_{m=0}^{p}$. An appropriate scaling results in a basis of the PUM space $S$ :

$$
\begin{aligned}
b_{1, m}(t) & :=\varphi_{1}(t) t^{2} P_{m-2}\left(\frac{2}{t_{1}} t-1\right) \quad m=2, \ldots, \max (2, p), \\
b_{i, m}(t) & :=\varphi_{i}(t) P_{m}\left(2 \frac{t-t_{i-2}}{t_{i}-t_{i-2}}-1\right) \quad m=0, \ldots, p, i=2, \ldots, N-1, \\
b_{N, m}(t) & :=\varphi_{N}(t) P_{m}\left(2 \frac{t-t_{N-2}}{t_{N-1}-t_{N-2}}-1\right) \quad m=0, \ldots, p .
\end{aligned}
$$

Figure 2 shows the shape of these basis functions for some different values of $m$ on a non-uniform time grid. For $m=0$ the basis functions are simply the shape functions of the partition of unity. For higher $m$ this function is multiplied by the appropriate Legendre polynomial.

\section{Properties of the temporal basis functions}

In this section we investigate the growth behaviour of the $k$ th derivative of the bump functions $\rho_{a, b, c}$ which were introduced in the last section. In the context of a Galerkin discretization, an important property of these functions and functions which are composed of them is, whether they allow for a fast numerical integration. In this light, we analyze the error that arises from approximating integrals of the form 


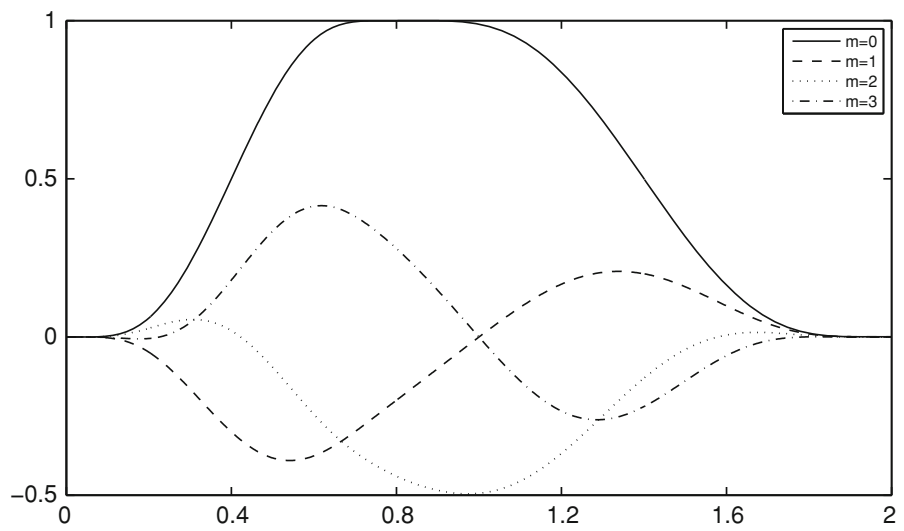

Fig. 2 Basis function of $S$ for $t_{0}=0, t_{1}=0.8$ and $t_{2}=2$

$$
\mathcal{I} \rho_{a, b, c}:=\int_{a}^{c} \rho_{a, b, c}(t) d t=\frac{c-a}{2}
$$

by a $n$-point Gauss-Legendre quadrature rule in the interval $[a, c]$, denoted by $Q_{n} \rho_{a, b, c}$. We carry out the quadrature error analysis for these simple integrals in quite some detail by using the derived growth estimates of our bump functions and its derivatives. We consider this analysis as an important first step in order to estimate the quadrature error for integrals of the form (3.4) using tensorized Gauss quadrature rules. Note, however, that for the full space-time integrals the singularity at $r=\|x-y\|=0$ has to be taken into account in the spatial part of the quadrature method, e.g., by using regularizing coordinates (cf. [24]). An analysis of the arising quadrature error for the full space-time integral is still an open question and we expect that it can be based on the results derived in this section.

Recall that for given $a<b<c \in \mathbb{R}$, the functions $\rho_{a, b, c}(t)$ are $C^{\infty}$-bump functions with supp $\rho_{a, b, c}=[a, c]$. It is well known that Gauss-Legendre quadrature converges exponentially for integrands that are analytic in a sufficiently large (complex) neighborhood of the integration domain. Since the functions $\rho_{a, b, c}(t)$ are smooth but not analytic in the points $a, b$ and $c$, these classical estimates for the quadrature error

$$
\mathcal{E}_{n} \rho_{a, b, c}:=\left|\mathcal{I} \rho_{a, b, c}-Q_{n} \rho_{a, b, c}\right|
$$

do not hold. We define the linear scaling functions

$$
\begin{aligned}
& \zeta_{r, s}:[r, s] \rightarrow[-1,1], \quad t \mapsto 2 \frac{t-r}{s-r}-1 \text { and its inverse } \\
& \xi_{r, s}:[-1,1] \rightarrow[r, s], \quad t \mapsto \frac{1}{2}(s-r)(t+1)+r .
\end{aligned}
$$

In order to find bounds for $\mathcal{E}_{n} \rho_{a, b, c}$ we need the following lemma. 
Lemma 4.1 Let $n \in \mathbb{N}$ and $1 \leq k \leq 2 n$. Then we have for $g \in C^{k+1}([a, b])$,

$$
\left|\mathcal{I} g-Q_{n} g\right| \leq \frac{32}{15 \pi}\left(\frac{b-a}{2}\right)^{k+1} \frac{1}{k(2 n+1-k)^{k}} \int_{a}^{b} \frac{\left|g^{(k+1)}(t)\right|}{\sqrt{1-\zeta_{a, b}(t)^{2}}} d t .
$$

Proof For the interval $[a, b]=[-1,1]$, Theorem 4.5 in [28] gives

$$
\left|\mathcal{I} g-Q_{n} g\right| \leq \frac{32}{15 \pi} \frac{1}{k(2 n+1-k)^{k}} \int_{-1}^{1} \frac{\left|g^{(k+1)}(t)\right|}{\sqrt{1-t^{2}}} d t
$$

for $k \in\{1, \ldots, 2 n\}$. For general $[a, b]$, a linear change of variable leads to

$$
\left|\mathcal{I} g-Q_{n} g\right| \leq \frac{32}{15 \pi}\left(\frac{b-a}{2}\right)^{k+2} \frac{1}{k(2 n+1-k)^{k}} \int_{-1}^{1} \frac{\left|g^{(k+1)}\left(\xi_{a, b}(t)\right)\right|}{\sqrt{1-t^{2}}} d t
$$

The substitution $t=\zeta_{a, b}(t)$ leads to the desired result.

In our case Lemma 4.1 reads

$$
\mathcal{E}_{n} \rho_{a, b, c} \leq \frac{32}{15 \pi}\left(\frac{c-a}{2}\right)^{k+1} \frac{1}{k(2 n+1-k)^{k}} \int_{a}^{c} \frac{\left|\rho_{a, b, c}^{(k+1)}(t)\right|}{\sqrt{1-\zeta_{a, c}(t)^{2}}} d t
$$

The definition of $\rho_{a, b, c}(t)$ leads to

$$
\begin{aligned}
\mathcal{E}_{n} \rho_{a, b, c} \leq & \frac{32}{15 \pi}\left(\frac{c-a}{2}\right)^{k+1} \frac{1}{k(2 n+1-k)^{k}} \\
& \times\left(\int_{a}^{b} \frac{\left|h_{a, b}^{(k+1)}(t)\right|}{\sqrt{1-\zeta_{a, c}(t)^{2}}} d t+\int_{b}^{c} \frac{\left|h_{b, c}^{(k+1)}(t)\right|}{\sqrt{1-\zeta_{a, c}(t)^{2}}} d t\right) .
\end{aligned}
$$

The formula above shows that we have to estimate the derivatives of the cutoff functions $h_{a, b}$ and $h_{b, c}$.

Lemma 4.2 The cutoff function $h_{a, b}$ satisfies the estimate

$$
\left|h_{a, b}^{(k+1)}(t)\right| \leq \frac{C_{2}}{b-a}\left(\frac{2 C_{1}}{b-a}\right)^{k} k !\left|\frac{e^{-2 \operatorname{arctanh}^{2}\left(\zeta_{a, b}(t)\right)}}{\left(1-\zeta_{a, b}(t)^{2}\right)^{k+1}}\right| q^{k}\left(\zeta_{a, b}(t)\right)
$$

for $k \geq 1$ with $q(t):=\ln \frac{4}{1-t^{2}}, C_{1}:=6 \sqrt{2} e$ and $C_{2}=\frac{10 \kappa}{\sqrt{\pi}} \frac{C_{1} \ln (4)}{C_{1} \ln (4)-2}$ where $\kappa \approx$ 1.086435 .

Proof Use Theorem 8.4 and the chain rule. 
Further estimation of the bound in Lemma 4.2 leads to:

Lemma 4.3 Let $q(x), C_{1}$ and $C_{2}$ be as in Lemma 4.2. Then we have

$$
\left\|h_{a, b}^{(k+1)}\right\|_{\infty} \leq \frac{C_{2}}{b-a}\left(\frac{2 \lambda 4^{1 / \lambda} C_{1}}{b-a}\right)^{k} k ! e^{\sigma_{(1+1 / \lambda) k+1}}
$$

for $k \geq 1$ and $\lambda>0$, where

$$
\sigma_{\alpha}:=\frac{1}{4} \alpha^{2}+\frac{1}{2}-\ln \left(\frac{1}{2} \alpha+\frac{1}{2} \sqrt{\alpha^{2}-4}\right)
$$

for $\alpha \geq 2$.

Proof Since

$$
q^{k}(x)=\left(\ln \frac{4}{1-x^{2}}\right)^{k} \leq\left(4^{1 / \lambda} \lambda\right)^{k}\left(\frac{1}{1-x^{2}}\right)^{k / \lambda}
$$

for $\lambda>0$, the result follows from Lemmas 4.2 and 8.5.

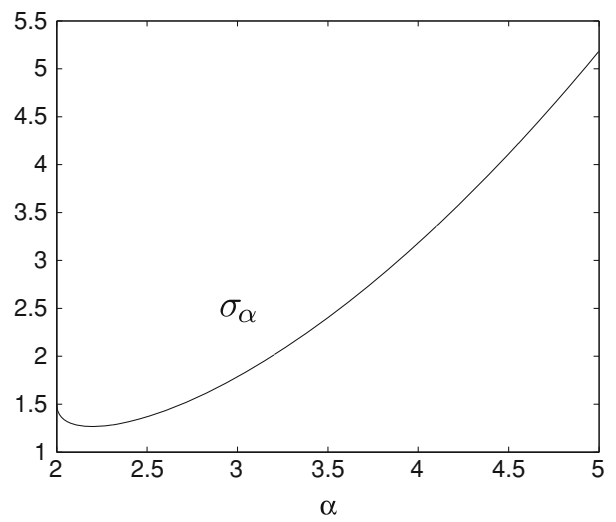

Corollary 4.4 The bump function $\rho_{a, b, c}$ satisfies the estimate

$$
\begin{aligned}
\left\|\rho_{a, b, c}^{(k+1)}\right\|_{\infty} \leq\left\|h_{a, b}^{(k+1)}\right\|_{\infty} \quad \text { in the case } b-a \leq c-b \\
\left\|\rho_{a, b, c}^{(k+1)}\right\|_{\infty} \leq\left\|h_{b, c}^{(k+1)}\right\|_{\infty} \quad \text { in the case } c-b \leq b-a .
\end{aligned}
$$

In order to estimate $\mathcal{E}_{n} \rho_{a, b, c}$, we assume that $b-a \leq c-b$, the other case being treated analogously. Furthermore we assume $c-a \leq c_{\max }(b-a)$, which corresponds to the local quasiuniformity of a given time mesh. With

$$
\frac{1}{\sqrt{1-\zeta_{a, c}(t)^{2}}} \leq \frac{c_{\max }}{\sqrt{1-\zeta_{a, b}(t)^{2}}}, \quad t \in(a, b),
$$


Lemmas 4.2, 8.5, and 8.6 we get

$$
\begin{aligned}
\int_{a}^{b} \frac{\left|h_{a, b}^{(k+1)}(t)\right|}{\sqrt{1-\zeta_{a, c}(t)^{2}}} d t & \leq \frac{C_{2}}{b-a}\left(\frac{2 C_{1}}{b-a}\right)^{k} k ! \int_{a}^{b} \frac{\left|\mathrm{e}^{-2 \operatorname{arctanh}^{2}\left(\zeta_{a, b}(t)\right) \mid}\right| q^{k}\left(\zeta_{a, b}(t)\right)}{\left|\left(1-\zeta_{a, b}(t)^{2}\right)^{k+1}\right| \sqrt{1-\zeta_{a, c}(t)^{2}}} d t \\
& \leq \frac{C_{2} c_{\max }}{b-a}\left(\frac{2 C_{1}}{b-a}\right)^{k} k ! \int_{a}^{b} \frac{\mid \mathrm{e}^{-2 \operatorname{arctanh}^{2}\left(\zeta_{a, b}(t)\right) \mid q^{k}\left(\zeta_{a, b}(t)\right)}}{\left|\left(1-\zeta_{a, b}(t)^{2}\right)^{k+3 / 2}\right|} d t \\
& \leq \frac{C_{2} c_{\max }}{b-a}\left(\frac{2 C_{1}}{b-a}\right)^{k} k ! \mathrm{e}^{\sigma_{k+3 / 2}} \int_{a}^{b} q^{k}\left(\zeta_{a, b}(t)\right) d t \\
& \leq \frac{C_{2} c_{\max }}{2}\left(\frac{2 C_{1}}{b-a}\right)^{k} k ! \mathrm{e}^{\sigma_{k+3 / 2}} \int_{-1}^{1} q^{k}(t) d t \\
& \leq 8 C_{2} c_{\max }\left(\frac{2 C_{1}}{b-a}\right)^{k}(k !)^{2} \mathrm{e}^{\sigma_{k+3 / 2}},
\end{aligned}
$$

where $\sigma_{k+3 / 2}$ is as in Lemma 4.3. Similar arguments show that also

$$
\int_{b}^{c} \frac{\left|h_{b, c}^{(k+1)}(t)\right|}{\sqrt{1-\zeta_{a, c}(t)^{2}}} d t \leq 8 C_{2} c_{\max }\left(\frac{2 C_{1}}{b-a}\right)^{k}(k !)^{2} \mathrm{e}^{\sigma_{k+3 / 2}}
$$

holds. With (4.2) the quadrature error can be estimated by

$$
\mathcal{E}_{n} \rho_{a, b, c} \leq \frac{256 C_{2} c_{\max }(c-a)}{15 \pi} \frac{1}{k(2 n+1-k)^{k}}\left(C_{1} c_{\max }\right)^{k}(k !)^{2} \mathrm{e}^{\sigma_{k+3 / 2}}
$$

for $k \in\{1, \ldots, 2 n\}$. Finally, Stirling's estimate $k ! \leq 1.1 \sqrt{2 \pi k} k^{k} \mathrm{e}^{-k}$ yields

$$
\mathcal{E}_{n} \rho_{a, b, c} \leq 41.5 C_{2} c_{\max }(c-a)\left(\frac{C_{1} c_{\max } k^{2}}{(2 n+1-k) \mathrm{e}^{2}}\right)^{k} \mathrm{e}^{\sigma_{k+3 / 2}}
$$

for $k \in\{1, \ldots, 2 n\}$. It remains to choose $k$ such that the right-hand side in the above inequality becomes small. We define

$$
E_{c_{\max }}(n, k):=\left(\frac{C_{1} c_{\max } k^{2}}{(2 n+1-k) \mathrm{e}^{2}}\right)^{k} \mathrm{e}^{\sigma_{k+3 / 2}}
$$

for $k \in\{1, \ldots, 2 n\}$. The next lemma shows that $E_{c_{\max }}(n, k)$ decays superalgebraically for an appropriate choice of $k$. 
Lemma 4.5 Let $\gamma \in\left(0, \frac{3}{4}\right)$ and $a, b, c \in \mathbb{R}$ with $c-a \leq c_{\max }(b-a)$ be given. If $n \in \mathbb{N}_{\geq 3}$ satisfies the condition

$$
(\ln n)^{2} n^{-3 / 4+\gamma} \leq \frac{2-e^{-2}}{C_{1} c_{\max } e^{-1 / 4}}
$$

the error bound

$$
\mathcal{E}_{n} \rho_{a, b, c} \leq \hat{C} n^{-\gamma \ln (n)}
$$

holds, with $\hat{C}:=41.5 C_{2} c_{\max }(c-a)\left(2-e^{-2}\right) e^{17 / 16}$.

Proof Since

$$
\sigma_{k+3 / 2} \leq \frac{1}{4} k^{2}+\frac{3}{4} k+\frac{17}{16}
$$

we have

$$
E_{c_{\max }}(n, k) \leq \mathrm{e}^{17 / 16}(\tilde{C})^{k} k^{2 k}(2 n+1-k)^{-k} \mathrm{e}^{k^{2} / 4}
$$

where $\tilde{C}:=C_{1} c_{\max } \mathrm{e}^{-5 / 4}$. We set $k=\lfloor\ln (n)\rfloor$ and get

$$
\begin{aligned}
E_{c_{\max }}(n,\lfloor\ln (n)\rfloor) & \leq \mathrm{e}^{17 / 16}(\tilde{C})^{\lfloor\ln (n)\rfloor}\lfloor\ln (n)\rfloor^{2\lfloor\ln (n)\rfloor}(2 n+1-\lfloor\ln (n)\rfloor)^{-\lfloor\ln (n)\rfloor} \mathrm{e}^{\lfloor\ln (n)\rfloor^{2} / 4} \\
& \leq \mathrm{e}^{17 / 16}(\tilde{C})^{\ln (n)} \ln (n)^{2 \ln (n)}(2 n+1-\ln (n))^{-\ln (n)+1} \mathrm{e}^{(\ln n)^{2} / 4} .
\end{aligned}
$$

Simple calculus shows

$$
1-\ln (n) \geq-\mathrm{e}^{-2} n \text { for } n \in \mathbb{N},
$$

so that the error can be estimated by

$$
E_{c_{\text {max }}}(n,\lfloor\ln (n)\rfloor) \leq\left(2-\mathrm{e}^{-2}\right) \mathrm{e}^{17 / 16}\left(\frac{\tilde{C}}{2-\mathrm{e}^{-2}}\right)^{\ln (n)}(\ln n)^{2 \ln (n)} n^{-\ln (n)+1} \mathrm{e}^{\ln (n)^{2} / 4} .
$$

Applying the logarithm on both sides yields

$$
\begin{aligned}
\ln \left(E_{c_{\max }}(n,\lfloor\ln (n)\rfloor)\right) \leq & \ln \left(\left(2-\mathrm{e}^{-2}\right) \mathrm{e}^{17 / 16}\right) \\
& +\ln (n)\left[\ln \left(\frac{\tilde{C}}{2-\mathrm{e}^{-2}}\right)+2 \ln (\ln (n))+1-\frac{3}{4} \ln (n)\right] .
\end{aligned}
$$

For given $\gamma \in\left(0, \frac{3}{4}\right)$, let $n$ satisfy condition (4.3). Then we get

$$
\ln \left(E_{c_{\max }}(n,\lfloor\ln (n)\rfloor)\right) \leq \ln \left(\left(2-\mathrm{e}^{-2}\right) \mathrm{e}^{17 / 16}\right)-\gamma(\ln n)^{2},
$$

which leads to the desired result. 
Table $1 k_{\text {opt }}$ for different ranges of $n$ and different $c_{\max }$

\begin{tabular}{lllllll}
\hline$c_{\max }=2.0$ & $n$ & $2-680$ & $681-5,929$ & $5,930-33,776$ & $33,777-157,999$ & $158,000-659,277$ \\
& $k_{\text {opt }}$ & 1 & 2 & 3 & 4 & 5 \\
$c_{\max }=2.2$ & $n$ & $2-748$ & $749-6,522$ & $6,523-37,153$ & $37,154-173,799$ & $173,800-725,205$ \\
& $k_{\text {opt }}$ & 1 & 2 & 3 & 4 & 5 \\
$c_{\max }=2.4$ & $n$ & $2-816$ & $817-7,115$ & $7,116-40,531$ & $40,532-189,598$ & $189,599-791,132$ \\
& $k_{\text {opt }}$ & 1 & 2 & 3 & 4 & 5 \\
\hline
\end{tabular}

Table 2 Results for different choices of $c_{\max }, \delta$ and $r$

\begin{tabular}{llllll}
\hline$c_{\max }$ & $\delta$ & $r$ & $n_{\min }$ & $n_{\max }$ & $E_{c_{\max }}\left(n_{\max }, k_{\text {opt }}\right)$ \\
\hline 2.0 & 0.25 & 18 & 11 & 846,975 & $\approx 1.2 \times 10^{-12}$ \\
& 0.26 & 18 & 12 & 92,231 & $\approx 5.9 \times 10^{-8}$ \\
2.2 & 0.25 & 20 & 11 & 649,170 & $\approx 9.4 \times 10^{-12}$ \\
& 0.26 & 20 & 12 & 67,353 & $\approx 3.0 \times 10^{-7}$ \\
2.4 & 0.25 & 22 & 11 & 545,048 & $\approx 3.5 \times 10^{-11}$ \\
& 0.26 & 22 & 12 & 33,776 & $\approx 6.4 \times 10^{-6}$ \\
\hline
\end{tabular}

Remark 4.6 The asymptotic behaviour of the error bound in Lemma 4.5 is sharp in the sense that the choice $k=\left\lfloor(\ln n)^{\delta}\right\rfloor$ with $\delta>1$ leads to the divergence of $E_{c_{\max }}(n, k)$ if $n$ tends to infinity.

Although Lemma 4.5 suggests that the error of Gauss-Legendre quadrature applied to integrals of the form (4.1) decreases superalgebraically but not exponentially, we want to show numerically that $E_{c_{\max }}(n, k)$ decays faster for certain ranges of $n$. In order to demonstrate this, an appropriate choice of $k$ is crucial. Lemma 4.5 shows that $k$ has to be chosen very small compared to $n$ due to the fast growth of the derivatives of $\rho_{a, b, c}$. To illustrate this, Table 1 shows the optimal $k$, denoted by $k_{\text {opt }}$, such that $E_{c_{\max }}(n, k)$ is minimal for given $n$ and different $c_{\max }$.

Based on these observations we choose $k$ optimal for every $n$ and want to determine $r, \delta \in \mathbb{R}_{\geq 0}$ such that the estimate

$$
E_{c_{\max }}\left(n, k_{\mathrm{opt}}\right) \leq r \mathrm{e}^{-n^{\delta}}
$$

holds for a preferably large range $n_{\min } \leq n \leq n_{\max }$.

Table 2 shows the results of numerical experiments. It can be observed that $\mathcal{E}_{n} \rho_{a, b, c}=\mathcal{O}\left(\mathrm{e}^{-n^{1 / 4}}\right)$ for a large range of $n$ in the case $c_{\max } \in\{2.0,2.2,2.4\}$.

Figure 3 shows the decay of the error in the case of the bump function $\rho_{0, \frac{10}{11}, 2}$ which corresponds to $c_{\max }=2.2$. It can be observed that $\mathcal{E}_{n} \rho_{0, \frac{10}{11}, 2}$, which represents the relative error since $\mathcal{I} \rho_{0, \frac{10}{11}, 2}=1$, decays even faster than predicted by theory at least for those accuracies that are of interest in practical computations.

The influence of $c_{\max }$ is rather small in practice. Numerical tests show that the error behaviour is similar to the one in Fig. 3 for different (moderate) $c_{\max }$. 


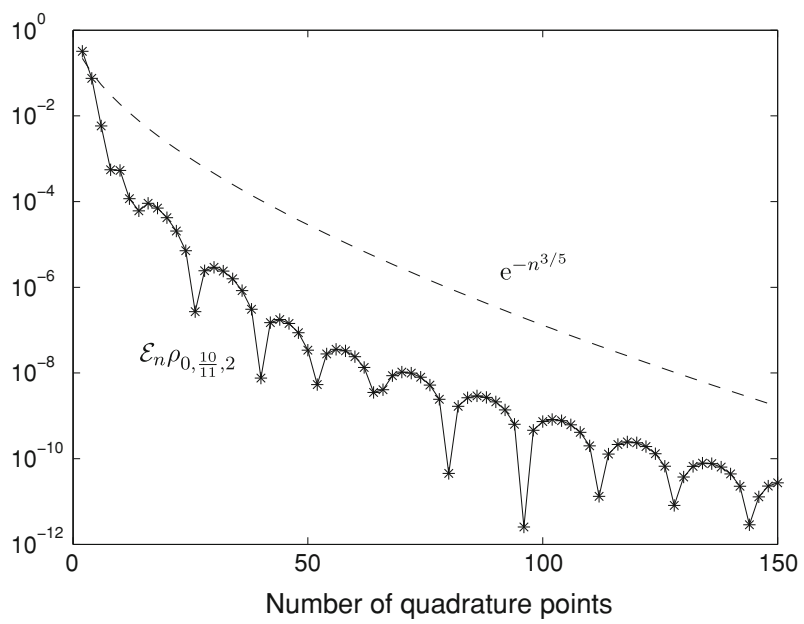

Fig. 3 Quadrature error for the case $c_{\max }=2.2$, i.e., we consider the bump function $\rho_{0, \frac{10}{11}, 2}$

\section{Application to a problem on the sphere}

In this section we apply a Galerkin method using our new basis functions in time to the integral equation (2.3) in the case where the boundary $\Gamma$ is the unit sphere $\mathbb{S}^{2}$. Furthermore we assume that the right-hand side $g$ is causal, i.e., $g(x, t)=0$ for $t \leq 0$ and that at least the first time derivative of $g$ vanishes at $t=0$. Moreover, $g$ is supposed to be of the form

$$
g(x, t)=g(t) Y_{n}^{m},
$$

where $Y_{n}^{m}$ denotes a spherical harmonic of degree $n$ and order $m$. This setting was already used in [6] and allows to reduce the boundary integral equation (2.3) to a univariate problem in time. To see this note that an equivalent formulation of the retarded single layer potential (2.2) is given by

$$
S \phi(x, t)=\int_{0}^{t} \int_{\Gamma} k(x-y, t-\tau) \phi(y, \tau) d \Gamma_{y} d \tau, \quad(x, t) \in \Omega \times[0, T],
$$

where $k(z, t)$ is the fundamental solution of the wave equation,

$$
k(z, t)=\frac{\delta(t-\|z\|)}{4 \pi\|z\|},
$$

$\delta(t)$ being the Dirac delta distribution. Furthermore we introduce the single layer potential for the Helmholtz operator $\Delta U-s^{2} U=0$ which is given by

$$
(V(s) \varphi)(x):=\int_{\Gamma} K(s, x-y) \varphi(y, \tau) d \Gamma_{y},
$$


where

$$
K(s, z):=\frac{\mathrm{e}^{-s\|z\|}}{4 \pi\|z\|}
$$

is the fundamental solution of the Helmholtz equation in three dimensions. An important property of the single layer potential $V(s)$ is that

$$
V(s) Y_{n}^{m}=\lambda_{n}(s) Y_{n}^{m},
$$

i.e., the spherical harmonics $Y_{n}^{m}$ are eigenfunctions of this operator with eigenvalues $\lambda_{n}(s)$. The latter can be expressed in terms of modified Bessel functions $I_{\kappa}$ and $K_{\kappa}$ (see [1])

$$
\lambda_{n}(s)=I_{n+\frac{1}{2}}(s) K_{n+\frac{1}{2}}(s) .
$$

Next, we will transform equation (2.3) into frequency domain using Laplace transformations. Property (5.2) and a back transformation then leads to a univariate problem in time. Recall the definition of the Laplace transform

$$
\hat{\phi}(s):=(\mathcal{L} \phi)(s)=\int_{0}^{\infty} \phi(t) \mathrm{e}^{-s t} d t
$$

with inverse

$$
\left(\mathcal{L}^{-1} \hat{\phi}\right)(s)=\frac{1}{2 \pi i} \int_{\sigma-i \infty}^{\sigma+i \infty} \hat{\phi}(s) \mathrm{e}^{s t} d s .
$$

Note that the fundamental solution of the Helmholtz equation is the Laplace transform of the fundamental solution of the wave equation. Using the representation (5.1) for $S$ and expressing $k$ in terms of its Laplace transform leads to the integral equation

$$
\begin{aligned}
g(t) Y_{n}^{m} & =\int_{0}^{t} \int_{\Gamma} k(t-\tau,\|x-y\|) \phi(y, \tau) d \Gamma_{y} d \tau \\
& =\frac{1}{2 \pi i} \int_{\sigma-i \infty}^{\sigma+i \infty} \int_{0}^{t} \mathrm{e}^{s \tau} \int_{\Gamma} K(s,\|x-y\|) \phi(y, t-\tau) d \Gamma_{y} d \tau d s \\
& =\frac{1}{2 \pi i} \int_{\sigma-i \infty}^{\sigma+i \infty} \int_{0}^{t} \mathrm{e}^{s \tau}(V(s) \phi(\cdot, t-\tau))(x) d \tau d s .
\end{aligned}
$$

Inserting the ansatz $\phi(x, t)=\phi(t) Y_{n}^{m}$ and using (5.2) leads to the one dimensional problem: find $\phi(t)$ such that 


$$
\int_{0}^{t} \mathcal{L}^{-1}\left(\lambda_{n}\right)(\tau) \phi(t-\tau) d \tau=g(t), \quad t \in[0, T]
$$

Note that $\phi(t) Y_{n}^{m}$ where $\phi(t)$ satisfies (5.4) is a solution of the full problem (2.1a)(2.1c) in the case where $\Gamma=\mathbb{S}^{2}$ and $g(x, t)=g(t) Y_{n}^{m}$. In order to analyse our new approach for the temporal discretization we choose (5.4) as our model problem.

Example Explicit representations of the exact solutions of (5.4) were computed in $[25,29]$.

(a) For $n=0$ the solution is given by

$$
\phi(t)=2 \sum_{k=0}^{\lfloor t / 2\rfloor} g^{\prime}(t-2 k)
$$

(b) For $n=1$ we have

$$
\begin{aligned}
\phi(t)= & 2 \sum_{k=0}^{\lfloor t / 2\rfloor}(-1)^{k} g^{\prime}(t-2 k)+2 \int_{0}^{t} \sinh (\tau) g^{\prime}(t-\tau) d \tau \\
& -2 \sum_{k=1}^{\lfloor t / 2\rfloor} \sum_{j=1}^{k} \int_{2 k}^{t}\left(c_{k, j}^{(2)}+c_{k, j}^{(3)} \tau-c_{k, j}^{(3)} 2 k\right)(\tau-2 k)^{j-1} \mathrm{e}^{\tau-2 k} g^{\prime}(t-\tau) d \tau,
\end{aligned}
$$

where

$$
\begin{aligned}
& c_{k, j}^{(2)}=(-1)^{k+1} \sum_{m=0}^{j-1} \frac{\left(1-(-1)^{j-m}\right) k !}{(j-1) ! m !(k-j) !(j-m) !} \quad \text { and } \\
& c_{k, j}^{(3)}=(-1)^{k+1} \frac{2^{j-1}(k-1) !}{(j-1) ! j !(k-j) !} .
\end{aligned}
$$

These formulas will serve as reference solutions for our numerical experiments.

In order to apply a Galerkin method to (5.4) we need a suitable variational formulation. If we choose $V_{\text {Galerkin }}$ in (3.2) by $V_{\text {Galerkin }}=Y_{n}^{m} S$, the space-time Galerkin discretization decouples and reduces to the purely temporal problem:

$$
\text { Find } \phi_{S} \in S: \quad \int_{0}^{T} \int_{0}^{t} \mathcal{L}^{-1}\left(\lambda_{n}\right)(\tau) \dot{\phi}_{S}(t-\tau) \zeta(t) d \tau d t=\int_{0}^{T} \dot{g}(t) \zeta(t) d t \quad \forall \zeta \in S \text {. }
$$

For the numerical solution of this equation we employ the representation with respect to the PUM basis (cf. (3.7)) and define the index set 


$$
\mathcal{P}_{i}:= \begin{cases}\{2,3, \ldots \max \{2, p\}\} & i=1 \\ \{0,1, \ldots, p\} & 2 \leq i \leq N\end{cases}
$$

Then, inserting the ansatz

$$
\phi_{S}(t)=\sum_{i=1}^{N} \sum_{m \in \mathcal{P}_{i}} \alpha_{i, m} b_{i, m}(t)
$$

leads to the discrete problem: find $\alpha_{i, m}$ such that

$$
\sum_{i=1}^{N} \sum_{m \in \mathcal{P}_{i}} \alpha_{i, m} \int_{0}^{T} \int_{0}^{t} \mathcal{L}^{-1}\left(\lambda_{n}\right)(t-\tau) \dot{b}_{i, m}(\tau) b_{j, k}(t) d \tau d t=\int_{0}^{T} \dot{g}(t) b_{j, k}(t) d t
$$

for $j=1,2, \ldots, N$ and $k \in \mathcal{P}_{j}$. In order to find the solution of (5.8) we have to compute $\mathcal{L}^{-1}\left(\lambda_{n}\right)(t)$. After some algebraic manipulations (cf. [25]) we obtain from (5.3)

$$
\lambda_{n}(s)=\sum_{l=0}^{2 n} \frac{c_{n, l}^{\mathrm{I}}}{s^{l+1}}+\mathrm{e}^{-2 s} \sum_{l=0}^{2 n} \frac{c_{n, l}^{\mathrm{II}}}{s^{l+1}}
$$

where

$$
c_{n, l}^{\mathrm{I}}:= \begin{cases}\sum_{j=0}^{l} \frac{1}{2}(-1)^{l-j}(n, l-j)(n, j), & \text { for } l \leq n, \\ \sum_{j=l-n}^{n} \frac{1}{2}(-1)^{l-j}(n, l-j)(n, j), & \text { for } n<l \leq 2 n\end{cases}
$$

and

$$
c_{n, l}^{\mathrm{II}}:= \begin{cases}\sum_{j=0}^{l} \frac{1}{2}(-1)^{n+1}(n, l-j)(n, j), & \text { for } l \leq n, \\ \sum_{j=l-n}^{n} \frac{1}{2}(-1)^{n+1}(n, l-j)(n, j), & \text { for } n<l \leq 2 n\end{cases}
$$

with $(n, k):=\frac{(n+k) !}{2^{k} k !(n-k) !}$. The inverse Laplace transform of $\lambda_{n}(s)$ is therefore given by

$$
\mathcal{L}^{-1}\left(\lambda_{n}\right)(t)=\sum_{l=0}^{2 n} \frac{c_{n, l}^{\mathrm{I}}}{l !} t^{l} H(t)+\sum_{l=0}^{2 n} \frac{c_{n, l}^{\mathrm{II}}}{l !}(t-2)^{l} H(t-2),
$$

where

$$
H(t)=\left\{\begin{array}{cc}
0 & t \leq 0 \\
1 & t>0
\end{array}\right.
$$


denotes the Heaviside step function. This shows that the discrete problem (5.8) is equivalent to: find $\alpha_{i, m}$ such that

$$
\begin{aligned}
& \sum_{i=1}^{N} \sum_{m \in \mathcal{P}_{i}} \alpha_{i, m}\left[\int_{0}^{T} \int_{0}^{t} q_{n}^{\mathrm{I}}(t-\tau) b_{i, m}(\tau) \dot{b}_{j, k}(t) d \tau d t\right. \\
& \left.\quad+\int_{0}^{T} \int_{0}^{t} q_{n}^{\mathrm{II}}(t-\tau-2) H(t-\tau-2) b_{i, m}(\tau) \dot{b}_{j, k}(t) d \tau d t\right]=\int_{0}^{T} g(t) \dot{b}_{j, k}(t) d t
\end{aligned}
$$

for $j=1, \ldots, N, k \in \mathcal{P}_{j}$, where

$$
q_{n}^{\mathrm{I}}(t):=\sum_{l=0}^{2 n} \frac{c_{n, l}^{\mathrm{I}}}{l !} t^{l} \quad \text { and } \quad q_{n}^{\mathrm{II}}(t):=\sum_{l=0}^{2 n} \frac{c_{n, l}^{\mathrm{II}}}{l !} t^{l}
$$

We now turn our attention to the numerical computation of the double integral

$$
\int_{0}^{T} \int_{0}^{t} q_{n}^{\mathrm{I}}(t-\tau) b_{i, m}(\tau) \dot{b}_{j, k}(t) d \tau d t
$$

arising in (5.9). Therefore let

$$
\begin{aligned}
\operatorname{supp} b_{i, m} & =\Theta_{i}=\left[m_{i}, M_{i}\right] \text { and } \\
\operatorname{supp} b_{j, k} & =\Theta_{j}=\left[m_{j}, M_{j}\right] .
\end{aligned}
$$

We write "..." short for " $q_{n}^{\mathrm{I}}(t-\tau) b_{i, m}(\tau) \dot{b}_{j, k}(t) d \tau d t$ " and distinguish between the following six cases (see Fig. 4):

(i) $m_{i} \leq M_{i} \leq m_{j} \leq M_{j}$. Then,

$$
\int_{0}^{T} \int_{0}^{t} \cdots=\int_{\Theta_{j}} \int_{\Theta_{i}} \cdots
$$

(ii) $m_{i} \leq m_{j} \leq M_{i} \leq M_{j}$. Then,

$$
\int_{0}^{T} \int_{0}^{t} \cdots=\int_{m_{j}}^{M_{i}} \int_{m_{i}}^{t} \cdots+\int_{M_{i}}^{M_{j}} \int_{\Theta_{i}} \cdots
$$




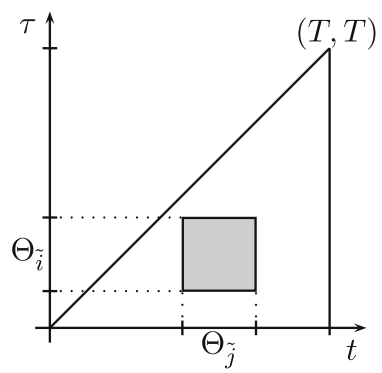

(a)

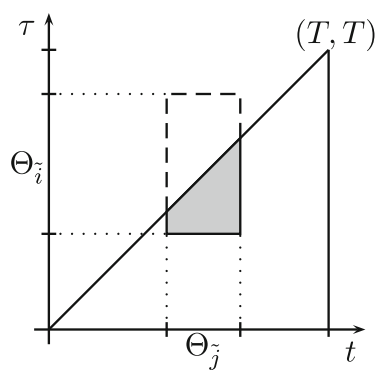

(d)

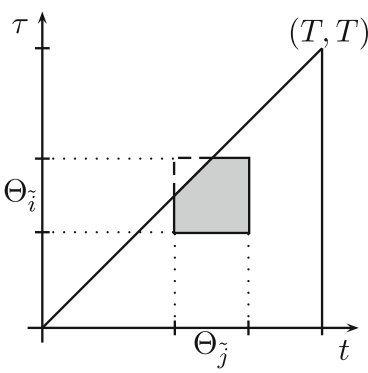

(b)

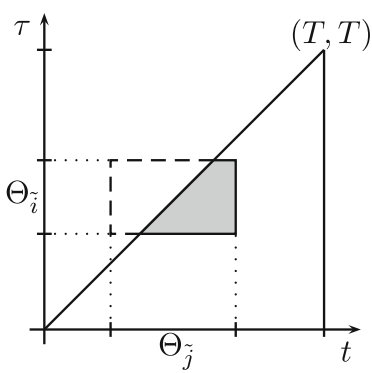

(e)

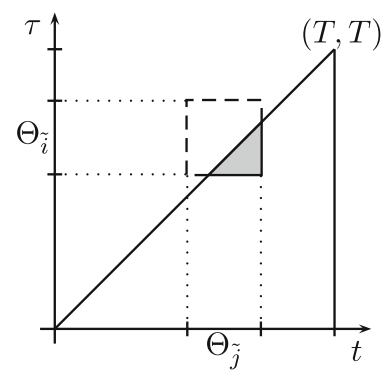

(c)

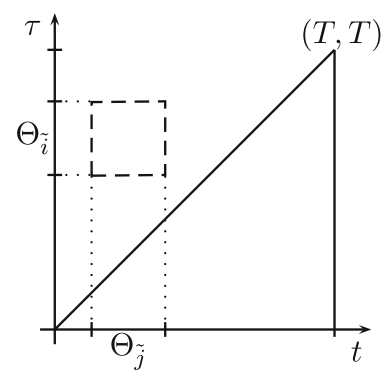

(f)

Fig. 4 Different domains of integration for integral (5.10). a Domain of integration case (i), b domain of integration case (ii), $\mathbf{c}$ domain of integration case (iii), $\mathbf{d}$ domain of integration case (iv), $\mathbf{e}$ domain of integration case (v), f domain of integration case (vi)

(iii) $m_{j} \leq m_{i} \leq M_{j} \leq M_{i}$. Then,

$$
\int_{0}^{T} \int_{0}^{t} \cdots=\int_{m_{i}}^{M_{j}} \int_{m_{i}}^{t} \cdots
$$

(iv) $m_{i} \leq m_{j} \leq M_{j} \leq M_{i}$. Then,

$$
\int_{0}^{T} \int_{0}^{t} \cdots=\int_{\Theta_{j}} \int_{m_{i}}^{t} \cdots
$$

(v) $m_{j} \leq m_{i} \leq M_{i} \leq M_{j}$. Then,

$$
\int_{0}^{T} \int_{0}^{t} \cdots=\int_{\Theta_{i}} \int_{m_{i}}^{t} \cdots+\int_{M_{i}}^{M_{j}} \int_{\Theta_{i}} \cdots
$$


(vi) $m_{j} \leq M_{j} \leq m_{i} \leq M_{i}$. Then,

$$
\int_{0}^{T} \int_{0}^{t} \cdots=0
$$

The computation of the second double integral

$$
\int_{0}^{T} \int_{0}^{t} q_{n}^{\mathrm{II}}(t-\tau-2) H(t-\tau-2) b_{i, m}(\tau) \dot{b}_{j, k}(t) d \tau d t
$$

in (5.9) is similar. Note that this integral vanishes for $T \leq 2$. For $T>2$ we have to distinguish between six cases as for the integrals in (5.10). We do not detail this here.

Remark 5.1 The resulting integration domains in the cases (i)-(vi) are either rectangles or triangles. Because simplex coordinates transform triangles to squares, we can restrict to rectangular integration domains and apply properly scaled $n$-point tensor GaussLegendre quadrature rules for the numerical approximation of the arising integrals.

\section{Numerical experiments}

In this section we present the results of numerical experiments. We solve the set of Eq. (5.9) in order to obtain a numerical solution of (5.4). The resulting error of the approximation, $\phi_{S}-\phi$, will be measured in the $L^{2}(0, T)$ norm. $L^{2}(0, T)$ is a suitable space for the solutions of (5.4) since it can be shown that if $\phi(t) \in L^{2}(0, T)$, then the corresponding solution of the full problem (2.3) satisfies $\phi(t) Y_{n}^{m} \in H^{-1 / 2,-1 / 2}(\Gamma \times$ $[0, T]) . \phi$ could also be considered in larger spaces than $L^{2}(0, T)$ but we expect analogous results of the numerical experiments in such spaces. We begin with the numerical tests and set

$$
g(t)= \begin{cases}t^{4} \mathrm{e}^{-2 t} & t \geq 0 \\ 0 & t<0\end{cases}
$$

In the following we check the sharpness of the convergence rates predicted by the theory in (3.6) for $n=0$ and $n=1$. We saw that the formulas for the exact solution of (5.4) involve derivatives of the right-hand side $g$ (cf. (5.5) and (5.6)). Since $g \in H^{4}(\mathbb{R})$ we therefore have $\phi \in H^{3}(\mathbb{R})$. Thus we expect a convergence rate with respect to the $L^{2}$-error of $h$ if we choose $p=0$, i.e., if we approximate simply by the shape functions of the partition of unity. We expect a convergence rate of $h^{2}$ if we choose $p=1$. These convergence rates could be confirmed by the numerical experiments (see Fig. 5).

Next, we investigate the behaviour of the method for a right-hand side that is less smooth:

$$
g(t)= \begin{cases}\sin ^{2}(2 t) \mathrm{e}^{-t} & t \geq 0 \\ 0 & t<0\end{cases}
$$




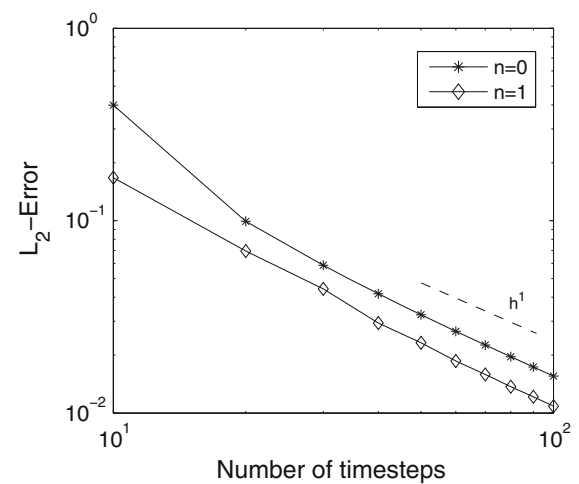

(a)

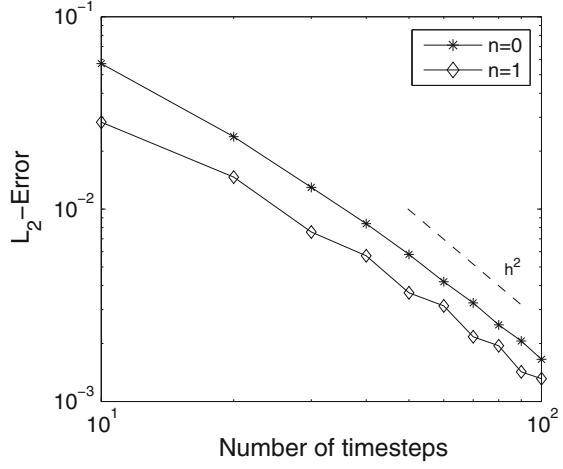

(b)

Fig. 5 Log- $\log$ scale plots of $\left\|\phi_{S}-\phi\right\|_{L^{2}([0, T])}$ for $T=6, g(t)=t^{4} \mathrm{e}^{-2 t}$ and $n$ as in (5.4). a Local polynomial approximation spaces of degree $p=0, \mathbf{b}$ local polynomial approximation spaces of degree $p=1$

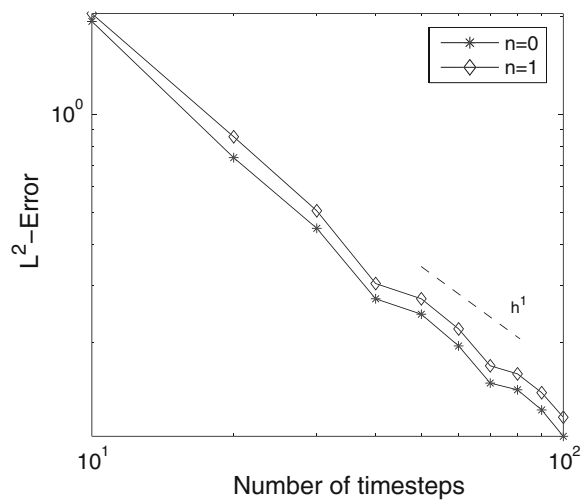

(a)

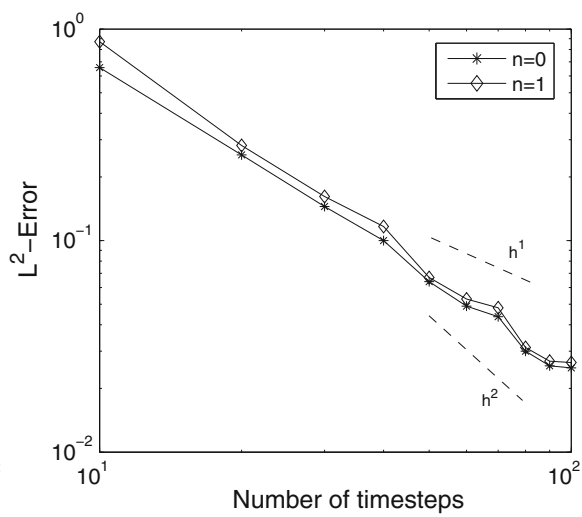

(b)

Fig. 6 Log-log scale plots of $\left\|\phi_{S}-\phi\right\|_{L^{2}([0, T])}$ for $T=6, g(t)=\sin ^{2}(2 t) \mathrm{e}^{-t}$ and $n$ as in (5.4). a Local polynomial approximation spaces of degree $p=0, \mathbf{b}$ local polynomial approximation spaces of degree $p=1$

Note that $g \in H^{2}(\mathbb{R})$ and therefore $\phi \in H^{1}(\mathbb{R})$. Hence we expect a convergence rate of $h$ in the case $p=0$. Due to the lack of smoothness of the solution we do not expect that higher order PUM spaces lead to better convergence rates. Indeed Fig. $6 \mathrm{~b}$ indicates that in the case $p=1$ a convergence rate of $h^{2}$ is not achieved.

The PUM with smooth basis functions (3.7) allows variable time steps which can be adapted to the smoothness, e.g., of the right-hand side. In the following we illustrate the benefit of this feature by a numerical example. We choose the right-hand side by

$$
g(t)= \begin{cases}-\sin (35 t) t^{3} \mathrm{e}^{-12(4 t-4)^{2}} & t \geq 0 \\ 0 & t<0\end{cases}
$$


Fig. $7 \quad g(t)=$

$-\sin (35 t) t^{3} \mathrm{e}^{-12(4 t-4)^{2}}$

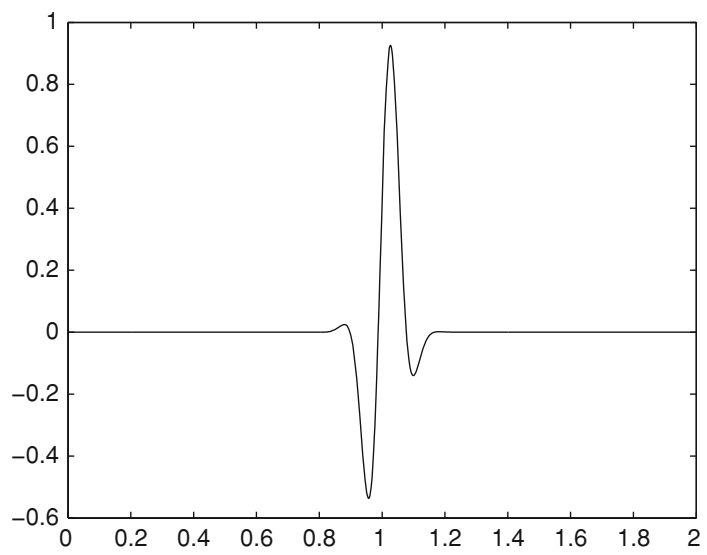

Fig. 8 Corresponding solution of (5.4) for $n=0$ and a time grid with variable meshwidth

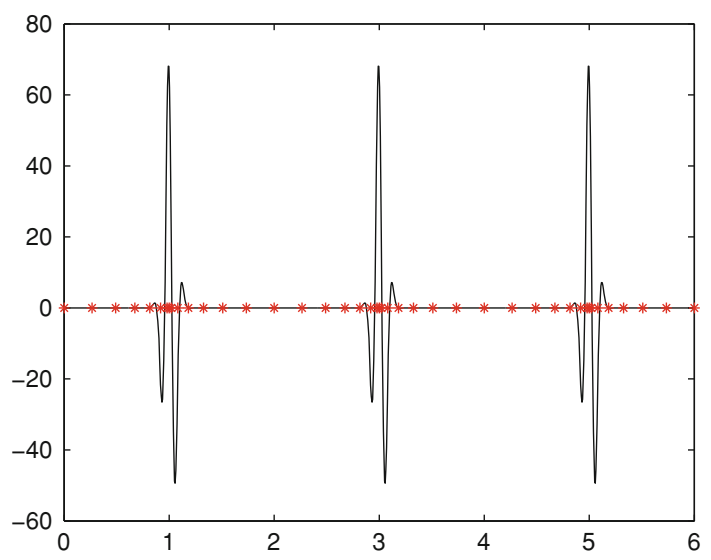

As we can see in Fig. 7 this function has a sharp pulse in the interval $\left(1-\frac{1}{5}, 1+\frac{1}{5}\right)$ and is almost zero otherwise. A similar behaviour can be observed for the corresponding solution $\phi$ for $n=0$. The 2-periodicity in (5.5) however implies that $\phi$ has peaks in small neighborhoods of all time points $t=2 l+1, l \in \mathbb{N}$ (cf. Fig. 8). Therefore we will employ a time mesh which is graded towards the time points $t=2 l+1$ where the solution is highly oscillatory. We use a quadratic grading of the uniformly distributed mesh points towards the origin:

$$
\pm\left(\frac{i}{m}\right)^{2} \quad 0 \leq i \leq m
$$

We number these mesh points from left to right $-1=\tilde{t}_{0}<\cdots<\tilde{t}_{2 m}=1$. Translation of these points to the time intervals $[2 l, 2 l+2]$ leads to the time mesh in Fig. 8.

Figure 9 shows the error plots for this right-hand side for $n=0$ and $p=1,2$. One can see that the error for the variable time mesh is considerably smaller than the error for the equidistant grid. Moreover the convergence starts earlier and the asymptotic 


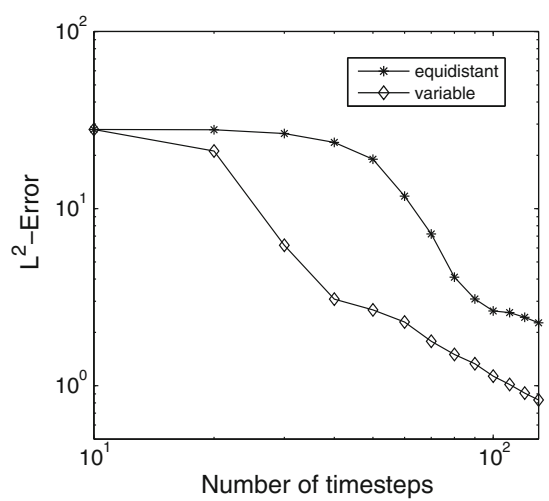

(a)

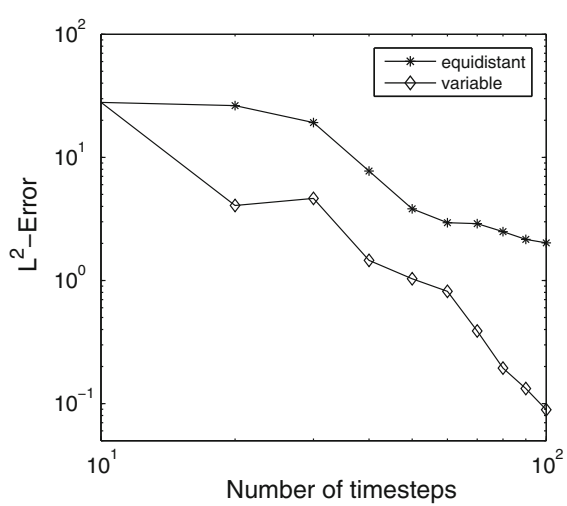

(b)

Fig. $9 \log -\log$ scale plots of $\left\|\phi_{S}-\phi\right\|_{L^{2}([0, T])}$ for $T=6, g(t)$ as in Fig. 7 and $n=0$. Comparison of equidistant and variable time meshes. a Local polynomial approximation spaces of degree $p=1$, b local polynomial approximation spaces of degree $p=2$

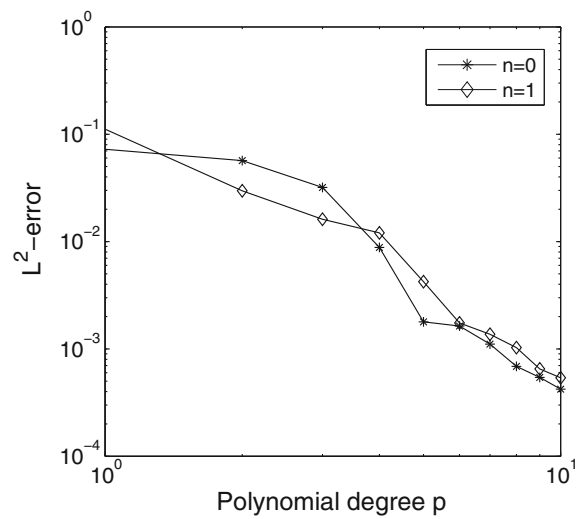

(a)

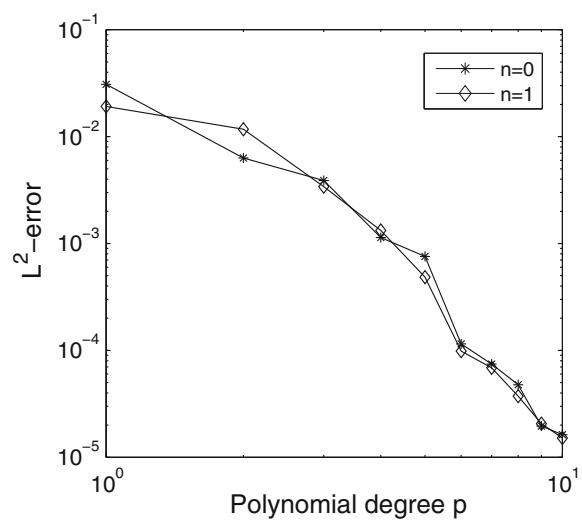

(b)

Fig. $10 \log -\log$ scale plots of $\left\|\phi_{S}-\phi\right\|_{L^{2}([0, T])}$ for $T=6, g(t)=t^{4} \mathrm{e}^{-2 t}$. a Number of timesteps: 5 , b number of timesteps: 10

convergence rate is already in the preasymptotic range. This shows that variable time stepping can improve the discretization substantially if knowledge about the solution is available. We expect similar benefits for the full problem.

Finally, we will show the performance of our method as a $p$-version for problems with smooth solutions, where we fix the number of timesteps and increase the polynomial degree of the local approximation spaces. Figure 10 shows two error plots for five and ten timesteps, where we again set $g(t)=t^{4} \mathrm{e}^{-2 t}$ for $t>0$. Recall that $g \in H^{4}(\mathbb{R})$ and therefore $\phi \in H^{3}(\mathbb{R})$. Thus the following error estimate holds (cf. [2]):

$$
\left\|\phi_{S}-\phi\right\|_{L^{2}([0,6])} \leq C p^{-2}\|\phi\|_{H^{3}([0,6])} \text {. }
$$




\section{Conclusion}

We have introduced a new set of basis functions in time for the discretization of retarded boundary integral formulations of the wave equation. The obtained basis functions are smooth, compactly supported, allow variable order of approximation and can be easily defined on an arbitrary time grid. In order to test the approach we applied a Galerkin method to a special case of the wave equation on the sphere for which analytic solutions are available. These solutions were used as reference solutions for the numerical experiments. It could be shown that the use of variable stepsizes in time can improve the convergence of the Galerkin scheme considerably provided that information about the behaviour of the solution is known in advance.

In a forthcoming paper we will apply this approach to the full problem, i.e., we will use a Galerkin method in space and time where we choose piecewise polynomial basis functions in space and our smooth PUM space in time in order to discretize the problem. The global smoothness of the basis function in time will simplify the computation of the entries of the boundary element matrix considerably since the numerical handling of the complicated geometry of the discrete light cone with the surface panels becomes superfluous - the use of curved surface panels becomes straightforward. Furthermore the boundary element matrix will be sparse due to the compact support of the basis functions.

Acknowledgments Thanks are due to Christoph Schwab for fruitful discussions concerning the use of the PUM for the time discretization.

\section{Appendix A: Technical estimates}

In this section we want to estimate the $n$th derivative of the function $f$ as defined in (3.5). Therefore let

$$
h(z):=\operatorname{erf}(z) \text { and } g(x):=\operatorname{arctanh} x=\frac{1}{2} \ln \frac{1+x}{1-x}
$$

such that $f:=h \circ 2 g$. Note that $[1,(7.1 .19)]$ implies

$$
h^{(n+1)}(z)=(-1)^{n} \frac{2}{\sqrt{\pi}} H_{n}(z) \mathrm{e}^{-z^{2}} \quad n=0,1,2, \ldots
$$

where $H_{n}$ are the Hermite polynomials. Hence,

$$
\begin{aligned}
f^{(n+1)}(x) & =\left(\frac{d}{d x}\right)^{n}\left(\frac{4}{\sqrt{\pi}\left(1-x^{2}\right)} \mathrm{e}^{-4 g^{2}(x)}\right) \\
& =\frac{4}{\sqrt{\pi}} \sum_{\ell=0}^{n}\left(\begin{array}{l}
n \\
\ell
\end{array}\right)\left(\frac{1}{1-x^{2}}\right)^{(\ell)}\left(\mathrm{e}^{-4 g^{2}(x)}\right)^{(n-\ell)} .
\end{aligned}
$$


Lemma 8.1 (Derivatives of $g$ ) It holds

$$
\left(\frac{1}{1-x^{2}}\right)^{(\ell)}=\frac{\ell ! p_{\ell}(x)}{\left(1-x^{2}\right)^{\ell+1}} \quad \forall x \in(-1,1),
$$

where

$$
p_{\ell}(x):=\frac{(x+1)^{\ell+1}-(x-1)^{\ell+1}}{2} .
$$

Furthermore, we have

$$
\left|g^{(\ell)}(x)\right| \leq\left\{\begin{array}{l}
\frac{1}{2} \ln \frac{4}{1-x^{2}} \quad \ell=0 \\
\frac{(\ell-1) ! 2^{\ell-1}}{\left(1-x^{2}\right)^{\ell}} \quad \ell \in \mathbb{N}_{\geq 1}
\end{array} \quad \forall x \in(-1,1)\right.
$$

as well as the more generous estimate

$$
\left|g^{(\ell)}(x)\right| \leq q(x) \frac{\ell ! 2^{\ell-1}}{\left(1-x^{2}\right)^{\ell}} \quad \forall \ell \in \mathbb{N}_{0}
$$

with $q(x)=\ln \frac{4}{1-x^{2}}$.

Lemma 8.2 (Derivative of composite functions) For $n \geq 1$ and $x \in(-1,1)$ we have

$$
\left(e^{-4 g^{2}(x)}\right)^{(n)}=e^{-4 g^{2}(x)} \sum_{k=1}^{n} A_{n, k}(x)(-1)^{k} H_{k}(2 g(x)),
$$

where

$$
A_{n, k}(x)=\frac{2^{k}}{k !} \sum_{\nu=1}^{k}(-1)^{k-v}\left(\begin{array}{l}
k \\
v
\end{array}\right) g^{k-v}(x)\left(g^{\nu}\right)^{(n)}(x)
$$

and

$$
\begin{aligned}
\left(g^{\nu}\right)^{(n)}= & \sum_{\ell_{v-1}=0}^{n} \sum_{\ell_{v-2}=0}^{\ell_{v-1}} \cdots \sum_{\ell_{1}=0}^{\ell_{2}}\left(\begin{array}{c}
n \\
\ell_{v-1}
\end{array}\right)\left(\begin{array}{l}
\ell_{v-1} \\
\ell_{n-2}
\end{array}\right) \cdots\left(\begin{array}{l}
\ell_{2} \\
\ell_{1}
\end{array}\right) g^{\left(n-\ell_{v-1}\right)} g^{\left(\ell_{v-1}-\ell_{v-2}\right)} \\
& \cdots g^{\left(\ell_{2}-\ell_{1}\right)} g^{\left(\ell_{1}\right)}
\end{aligned}
$$

Proof The representation (8.4a) and (8.4b) follows from [27, formulae (2), (7)], while (8.5) is proved by induction using Leibniz' product rule for differentiation. 
Lemma 8.3 (Estimate of derivatives of composite functions) For $n \geq 1$ and $x \in$ $(-1,1)$ we have

$$
\left|\left(e^{-g^{2}(x)}\right)^{(n)}\right| \leq \frac{5}{2} \kappa n ! e^{-2 g^{2}(x)}\left(\frac{C_{1} q(x)}{1-x^{2}}\right)^{n}
$$

with $\kappa \approx 1.086435$ and $C_{1}=6 \sqrt{2} e$.

Proof From (8.3) and (8.5) we conclude for all $n \geq 1, v \geq 1$, and $x \in(-1,1)$

$$
\begin{aligned}
\left|\left(g^{v}\right)^{(n)}(x)\right| & \leq n ! 2^{n-v} \frac{q^{v}(x)}{\left(1-x^{2}\right)^{n}} \sum_{\ell_{v-1}=0}^{n} \sum_{\ell_{v-2}=0}^{\ell_{v-1}} \cdots \sum_{\ell_{1}=0}^{\ell_{2}} 1 \\
& =n ! 2^{n-v} \frac{q^{v}(x)}{\left(1-x^{2}\right)^{n}}\left(\begin{array}{c}
n+v-1 \\
v-1
\end{array}\right) .
\end{aligned}
$$

Thus, from $(8.4 \mathrm{~b})$ we get that

$$
\begin{aligned}
\left|A_{n, k}(x)\right| & \leq \frac{2^{k} n !}{k !} \frac{q^{k}(x)}{\left(1-x^{2}\right)^{n}} \sum_{\nu=1}^{k}\left(\begin{array}{l}
k \\
v
\end{array}\right) 2^{n-v}\left(\begin{array}{c}
n+v-1 \\
v-1
\end{array}\right) \\
& \leq \frac{2^{n} n !}{k !} \frac{q^{k}(x)}{\left(1-x^{2}\right)^{n}}\left(\frac{n+k}{k}\right)^{k} \sum_{\nu=1}^{k}\left(\begin{array}{l}
k \\
v
\end{array}\right) 2^{k-v} \\
& \leq \frac{2^{n} n !}{k !} \frac{1}{\left(1-x^{2}\right)^{n}}\left(\frac{3(n+k) q(x)}{k}\right)^{k} .
\end{aligned}
$$

From [1, (22.14.17)] we obtain

$$
H_{k}(2 g(x)) \leq \mathrm{e}^{2 g^{2}(x)} \kappa 2^{k / 2} \sqrt{k !}
$$

The combination of (8.4a) and (8.4b), (8.5), (8.7) and (8.8) results in the estimate for the $n$th derivative of $\mathrm{e}^{-4 g^{2}(x)}$ :

$$
\begin{aligned}
\left|\left(\mathrm{e}^{-4 g^{2}(x)}\right)^{(n)}\right| & \leq \kappa 2^{n} n ! \frac{\mathrm{e}^{-2 g^{2}(x)}}{\left(1-x^{2}\right)^{n}} \sum_{k=1}^{n} \frac{1}{\sqrt{k !}}\left(\frac{3 \sqrt{2}(n+k) q(x)}{k}\right)^{k} \\
& \leq \kappa n ! \mathrm{e}^{-2 g^{2}(x)}\left(\frac{6 \sqrt{2} q(x)}{1-x^{2}}\right)^{n} \sum_{k=1}^{n} \frac{1}{\sqrt{k !}}\left(\frac{n+k}{k}\right)^{k} \\
& \leq \kappa n ! \mathrm{e}^{-2 g^{2}(x)}\left(\frac{6 \sqrt{2} \mathrm{e} q(x)}{1-x^{2}}\right)^{n} \sum_{k=1}^{n} \frac{1}{\sqrt{k !}} \\
& \leq \frac{5}{2} \kappa n ! \mathrm{e}^{-2 g^{2}(x)}\left(\frac{6 \sqrt{2} \mathrm{e} q(x)}{1-x^{2}}\right)^{n}
\end{aligned}
$$


Theorem 8.4 (Estimate of $n$th derivative of $f$ ) We have

$$
\left|f^{(n+1)}(x)\right| \leq C_{2} C_{1}^{n} n ! \frac{q(x)^{n}}{\left(1-x^{2}\right)^{n+1}} e^{-2 g^{2}(x)}
$$

with $C_{2}=\frac{10 \kappa}{\sqrt{\pi}} \frac{C_{1} \ln (4)}{C_{1} \ln (4)-2}$.

Proof From (8.1), (8.2) and (8.6) we get

$$
\begin{aligned}
\left|f^{(n+1)}(x)\right| & \leq \frac{10 \kappa}{\sqrt{\pi}} \sum_{l=0}^{n}\left(\begin{array}{l}
n \\
l
\end{array}\right) \frac{l ! 2^{l}}{\left(1-x^{2}\right)^{l+1}}(n-l) !\left(\frac{C_{1} q(x)}{1-x^{2}}\right)^{n-l} \mathrm{e}^{-2 g^{2}(x)} \\
& \leq \frac{10 \kappa}{\sqrt{\pi}} C_{1}^{n} n ! \frac{q(x)^{n}}{\left(1-x^{2}\right)^{n+1}} \mathrm{e}^{-2 g^{2}(x)} \sum_{l=0}^{n}\left(\frac{2}{C_{1} q(x)}\right)^{l} \\
& \leq \frac{10 \kappa}{\sqrt{\pi}} \frac{C_{1} \ln (4)}{C_{1} \ln (4)-2} C_{1}^{n} n ! \frac{q(x)^{n}}{\left(1-x^{2}\right)^{n+1}} \mathrm{e}^{-2 g^{2}(x)}
\end{aligned}
$$

which leads to the desired result.

Lemma 8.5 For $x \in(-1,1)$ and $\alpha \geq 2$, we have

$$
\left\|\frac{e^{-2 g^{2}(x)}}{\left(1-x^{2}\right)^{\alpha}}\right\|_{\infty} \leq e^{\sigma_{\alpha}}
$$

with

$$
\sigma_{\alpha}:=\frac{1}{4} \alpha^{2}+\frac{1}{2}-\ln \left(\frac{1}{2} \alpha+\frac{1}{2} \sqrt{\alpha^{2}-4}\right) .
$$

Proof We set

$$
\frac{\mathrm{e}^{-2 g^{2}(x)}}{\left(1-x^{2}\right)^{\alpha}}=\mathrm{e}^{s_{n}(x)},
$$

where

$$
s_{n}(x):=-2 \operatorname{arctanh}(x)^{2}-\alpha \ln \left(1-x^{2}\right) .
$$

With the definition of $\operatorname{arctanh}(x)$ we get

$$
\begin{aligned}
s_{n}(x)= & -2\left[\frac{1}{2} \ln (1+x)-\frac{1}{2} \ln (1-x)\right]^{2}-\alpha \ln (1-x)-\alpha \ln (1+x) \\
= & -\frac{1}{2}[\ln (1+x)]^{2}+\ln (1+x) \ln (1-x)-\frac{1}{2}[\ln (1-x)]^{2} \\
& -\alpha \ln (1-x)-\alpha \ln (1+x) .
\end{aligned}
$$


Since $s_{n}(x)$ is symmetric we assume $0 \leq x<1$ and get

$$
s_{n}(x) \leq-\frac{1}{2}[\ln (1-x)]^{2}-\alpha \ln (1-x)+\ln (1+x) \ln (1-x)=: \tilde{s}_{n}(x)
$$

$\tilde{s}_{n}(x)$ is strictly increasing in the interval $[0,0.5]$ for arbitrary $\alpha \in \mathbb{R}_{\geq 2}$. Therefore we may restrict to find an upper bound for $\tilde{s}_{n}(x)$ in the interval $[0.5,1[$. With the inequality $\ln (1+x) \ln (1-x) \leq-\ln (-\ln (1-x))$ we get

$$
\tilde{s}_{n}(x) \leq-\frac{1}{2}[\ln (1-x)]^{2}-\alpha \ln (1-x)-\ln (-\ln (1-x))=: \hat{s}_{n}(x)
$$

in $\left[0.5,1\left[\right.\right.$. The derivative of $\hat{s}_{n}(x)$ is given by

$$
\hat{s}_{n}^{\prime}(x)=\frac{[\ln (1-x)]^{2}+\alpha \ln (1-x)+1}{(1-x) \ln (1-x)}
$$

which has the root

$$
x_{0}=1-\mathrm{e}^{-\theta_{\alpha}},
$$

where $\theta_{\alpha}:=\frac{1}{2} \alpha+\frac{1}{2} \sqrt{\alpha^{2}-4}$. Inserting this above shows that

$$
s_{n}(x) \leq \alpha \theta_{\alpha}-\frac{1}{2} \theta_{\alpha}^{2}-\ln \theta_{\alpha}
$$

which leads to the desired result after some straightforward manipulations.

\section{Lemma 8.6 It holds}

$$
\int_{-1}^{1}\left(\ln \frac{4}{1-t^{2}}\right)^{n} d t \leq 16 n !
$$

for $n \in \mathbb{N}$.

Proof We first note that

$$
\begin{aligned}
& \int_{-1}^{1}|\ln (1-t)|^{i}|\ln (1+t)|^{k-i} d t \\
& \quad=\int_{-1}^{0}|\ln (1-x)|^{i}|\ln (1+t)|^{k-i} d t+\int_{0}^{1}|\ln (1-x)|^{i}|\ln (1+t)|^{k-i} d t \\
& \leq(\ln 2)^{i} \int_{-1}^{0}|\ln (1+t)|^{k-i} d t+(\ln 2)^{k-i} \int_{0}^{1}|\ln (1-t)|^{i} d t
\end{aligned}
$$




$$
\begin{aligned}
& =(\ln 2)^{i} \int_{0}^{1}|\ln (t)|^{k-i} d t+(\ln 2)^{k-i} \int_{0}^{1}|\ln (t)|^{i} d t \\
& =(\ln 2)^{i}(k-i) !+(\ln 2)^{k-i} i !,
\end{aligned}
$$

where we used $[17,(2.711)]$ in the last step. With these computations we get

$$
\begin{aligned}
\int_{-1}^{1}\left|\left(\ln \frac{4}{1-t^{2}}\right)^{n}\right| d t & \leq \sum_{k=0}^{n}\left(\begin{array}{l}
n \\
k
\end{array}\right) \int_{-1}^{1}\left|\ln \left(1-t^{2}\right)\right|^{k}(\ln 4)^{n-k} d t \\
& \leq \sum_{k=0}^{n} \sum_{i=0}^{k}\left(\begin{array}{l}
n \\
k
\end{array}\right)\left(\begin{array}{l}
k \\
i
\end{array}\right)(\ln 4)^{n-k} \int_{-1}^{1}|\ln (1-t)|^{i}|\ln (1+t)|^{k-i} d t \\
& \leq \sum_{k=0}^{n} \sum_{i=0}^{k}\left(\begin{array}{l}
n \\
k
\end{array}\right)\left(\begin{array}{l}
k \\
i
\end{array}\right)(\ln 4)^{n-k}\left((\ln 2)^{i}(k-i) !+(\ln 2)^{k-i} i !\right) \\
& \leq \sum_{k=0}^{n}\left(\begin{array}{l}
n \\
k
\end{array}\right)(\ln 4)^{n-k}\left(k ! \sum_{i=0}^{k} \frac{(\ln 2)^{i}}{i !}+k ! \sum_{i=0}^{k} \frac{(\ln 2)^{k-i}}{(k-i) !}\right) \\
& \leq 4 \sum_{k=0}^{n}\left(\begin{array}{l}
n \\
k
\end{array}\right)(\ln 4)^{n-k} k ! \\
& \leq 4 n ! \sum_{k=0}^{n} \frac{(\ln 4)^{n-k}}{(n-k) !} \leq 16 n !
\end{aligned}
$$

\section{References}

1. Abramowitz, M., Stegun, I.: Handbook of Mathematical Functions. Applied Mathematics Series, vol. 55. National Bureau of Standards, U.S. Department of Commerce (1972)

2. Babuška, I., Melenk, J.: The partition of unity method. Int. J. Numer. Methods Eng. 40, 727-758 (1997)

3. Bamberger, A., Ha Duong, T.: Formulation Variationnelle Espace-Temps pur le Calcul par Potientiel Retardé de la Diffraction d'une Onde Acoustique. Math. Methods Appl. Sci. 8, 405-435 (1986)

4. Banjai, L.: Multistep and multistage convolution quadrature for the wave equation: algorithms and experiments. SIAM J. Sci. Comput. 32(5), 2964-2994 (2010)

5. Banjai, L., Melenk, J., Lubich, C.: Runge-Kutta convolution quadrature for operators arising in wave propagation. Numer. Math. 119(1), 1-20 (2011)

6. Banjai, L., Sauter, S.: Rapid solution of the wave equation in unbounded domains. SIAM J. Numer. Anal. 47, 227-249 (2008)

7. Banjai, L., Schanz, M.: Wave propagation problems treated with convolution quadrature and BEM. Preprint 60/2010, MPI Leipzig.

8. Birgisson, B., Siebrits, E., Peirce, A.: Elastodynamic direct boundary element methods with enhanced numerical stability properties. Int. J. Numer. Methods Eng. 46, 871-888 (1999)

9. Bluck, M., Walker, S.: Analysis of three dimensional transient acoustic wave propagation using the boundary integral equation method. Int. J. Numer. Methods Eng. 39, 1419-1431 (1996) 
10. Chen, Q., Monk, P., Wang, X., Weile, D.: Analysis of convolution quadrature applied to the time-domain electric field integral equation (2012, submitted)

11. Chernov, A., von Petersdorff, T., Schwab, C.: Exponential convergence of hp quadrature for integral operators with Gevrey kernels. ESAIM Math. Model. Numer. Anal. (M2AN) 45(3), 387-422 (2011)

12. Davies, P., Duncan, D.: Averaging techniques for time-marching schemes for retarded potential integral equations. Appl. Numer. Math. 23, 291-310 (May 1997)

13. Davies, P., Duncan, D.: Numerical stability of collocation schemes for time domain boundary integral equations. In: Carstensen, C. (ed.) Computational Electromagnetics, pp. 51-86. Springer, Berlin (2003)

14. Ding, Y., Forestier, A., Ha Duong, T.: A Galerkin scheme for the time domain integral equation of acoustic scattering from a hard surface. J. Acoust. Soc. Am. 86(4), 1566-1572 (1989)

15. Dodson, S., Walker, S., Bluck, M.: Implicitness and stability of time domain integral equation scattering analysis. ACES J. 13, 291-301 (1997)

16. El Gharib, J.: Problèmes de potentiels retardés pour l'acoustique. PhD thesis, École Polytechnique (1999)

17. Gradshteyn, I.: Table of Integrals, Series, and Products. Academic Press, New York (1965)

18. Ha-Duong, T.: On retarded potential boundary integral equations and their discretisation. In: Topics in Computational Wave Propagation: Direct and Inverse Problems. Lecture Notes in Engineering and Computer Science, vol. 31, pp. 301-336. Springer, Berlin (2003)

19. Ha-Duong, T., Ludwig, B., Terrasse, I.: A Galerkin BEM for transient acoustic scattering by an absorbing obstacle. Int. J. Numer. Methods Eng. 57, 1845-1882 (2003)

20. Hackbusch, W., Kress, W., Sauter, S.: Sparse convolution quadrature for time domain boundary integral formulations of the wave equation by cutoff and panel-clustering. In: Schanz, M., Steinbach, O. (eds.) Boundary Element Analysis, pp. 113-134. Springer, Berlin (2007)

21. Hackbusch, W., Kress, W., Sauter, S.: Sparse convolution quadrature for time domain boundary integral formulations of the wave equation. IMA J. Numer. Anal. 29, 158-179 (2009)

22. Nédélec, J.C., Abboud, T., Volakis, J.: Stable solution of the retarded potential equations. In: Applied Computational Electromagnetics Society (ACES) Symposium Digest, 17th Annual Review of Progress, Monterey (2001)

23. Rynne, B., Smith, P.: Stability of time marching algorithms for the electric field integral equation. J. Electromagn. Waves Appl. 4, 1181-1205 (1990)

24. Sauter, S., Schwab, C.: Boundary Element Methods. Springer Series in Computational Mathematics. Springer, Berlin (2010)

25. Sauter, S., Veit, A.: A Galerkin method for retarded boundary integral equations with smooth and compactly supported temporal basis functions. Part II: implementation and reference solutions. Preprint 03-2011, Universität Zürich

26. Stephan, E., Maischak, M., Ostermann, E.: Transient boundary element method and numerical evaluation of retarded potentials. In: Computational Science-ICCS 2008, vol. 5102, pp. 321-330. Springer, Berlin (2008)

27. Todorov, P.: New explicit formulas for the $n$th derivative of composite functions. Pac. J. Math. 92(1), 217-236 (1981)

28. Trefethen, L.: Is Gauss quadrature better than Clenshaw-Curtis? SIAM Rev. 50, 67-87 (February 2008)

29. Veit, A.: A MATLAB code for computing exact solutions of retarded potential equations for a spherical scatterer (2011). https://www.math.uzh.ch/compmath/?exactsolutions

30. Wang, X., Wildman, R., Weile, D., Monk, P.: A finite difference delay modeling approach to the discretization of the time domain integral equations of electromagnetics. IEEE Trans. Antennas Propag. 56(8), 2442-2452 (2008)

31. Weile, D., Ergin, A., Shanker, B., Michielssen, E.: An accurate discretization scheme for the numerical solution of time domain integral equations. IEEE Antennas Propag. Soc. Int. Symp. 2, 741-744 (2000)

32. Weile, D., Pisharody, G., Chen, N., Shanker, B., Michielssen, E.: A novel scheme for the solution of the time-domain integral equations of electromagnetics. IEEE Trans. Antennas Propag. 52, 283-295 (2004)

33. Weile, D., Shanker, B., Michielssen, E.: An accurate scheme for the numerical solution of the time domain electric field integral equation. IEEE Antennas Propag. Soc. Int. Symp. 4, 516-519 (2001)

34. Wildman, A., Pisharody, G., Weile, D., Balasubramaniam, S., Michielssen, E.: An accurate scheme for the solution of the time-domain integral equations of electromagnetics using higher order vector bases and bandlimited extrapolation. IEEE Trans. Antennas Propag. 52, 2973-2984 (2004) 